Research Article

\title{
Solution of Fractional Differential Equations Utilizing Symmetric Contraction
}

\author{
Aftab Hussain \\ Department of Mathematics, King Abdulaziz University, P.O. Box 80203, Jeddah 21589, Saudi Arabia \\ Correspondence should be addressed to Aftab Hussain; aniassuirathka@kau.edu.sa
}

Received 11 February 2021; Revised 8 March 2021; Accepted 4 April 2021; Published 22 May 2021

Academic Editor: Huseyin Isik

Copyright (c) 2021 Aftab Hussain. This is an open access article distributed under the Creative Commons Attribution License, which permits unrestricted use, distribution, and reproduction in any medium, provided the original work is properly cited.

The aim of this paper is to present another family of fractional symmetric $\alpha-\eta$-contractions and build up some new results for such contraction in the context of $\mathscr{F}$-metric space. The author derives some results for Suzuki-type contractions and orbitally $T$-complete and orbitally continuous mappings in $\mathscr{F}$-metric spaces. The inspiration of this paper is to observe the solution of fractional-order differential equation with one of the boundary conditions using fixed-point technique in $\mathscr{F}$-metric space.

\section{Preliminaries and Scope}

Fixed-point theory has been promoted by a few particular works in the most recent decades [1-3]. One of the intriguing methodologies was presented in Karapinar et al.'s work [4] which starts a thought of interpolative kind of contractions and sest up shiny new fixed-point results in partial metric space. Recently, Jleli and Samet [5] introduced a new generalization of metric space and named it as $\mathscr{F}$-metric space.

Definition 1 (see [5]). Let $\mathscr{F}$ be the set function $f:(0,+\infty) \longrightarrow(-\infty,+\infty)$ that meets the following conditions:

$\left(\mathscr{F}_{1}\right) f$ is nondecreasing; that is, for all $0<c<d$, it implies $f(c) \leq f(d)$.

$\left(\mathscr{F}_{2}\right)$ For each iteration $\left\{d_{n}\right\} \subset(0,+\infty)$, we have $\lim _{n \longrightarrow+\infty} d_{n}=0, \quad$ if and only if $\lim _{n \longrightarrow+\infty} f\left(d_{n}\right)=-\infty$.

The generalized notion of metric space is as follows.

Definition 2 (see [5]). Let $A \neq \varnothing$ with $D: A \times A \longrightarrow[0,+\infty)$ be a given mapping. Suppose that there exists $(f, \mu) \in \mathscr{F} \times$ $[0,+\infty)$ such that
$\left(D_{1}\right)(w, v) \in A \times A, D(w, v)=0 \Leftrightarrow w=v$.

$\left(D_{2}\right) D(w, v)=D(v, w)$ for all $(w, v) \in A \times A$.

$\left(D_{3}\right)$ Each $(w, v) \in A \times A, \forall N \in \mathbb{N}, N \geq 2$, and for each $\left(u_{i}\right)_{i=1}^{N} \subset A$ with $\left(u_{1}, u_{N}\right)=(w, v)$, we have

$D(w, v)>0$ implies $f(D(w, v)) \leq f\left(\sum_{i=1}^{N-1} d\left(u_{i}, u_{i+1}\right)\right)+\mu$.

Then, it is said that $D$ is an $\mathscr{F}$-metric on $A$.

Here, the pair $(A, D)$ is called an $\mathscr{F}$-metric space and it is abbreviated as $\mathscr{F}$-MS. A sequence $\left\{w_{n}\right\}$ in $(A, D)$ is $\mathscr{F}$-Cauchy, if $\lim _{n, m \rightarrow \infty} D\left(w_{n}, w_{m}\right)=0$. Furthermore, $(A, D)$ is $\mathscr{F}$-complete, if every $\mathscr{F}$-Cauchy sequence is $\mathscr{F}$-convergent in $A$.

The following example is stated in [5].

Example 1 (see [5]). The set of natural numbers $\mathbb{N}=A$ is an $\mathscr{F}$-MS if we define $D$ by

$$
D(w, v)= \begin{cases}(w-v)^{2}, & \text { if }(w, v) \in[0,3] \times[0,3], \\ |w-v|, & \text { if }(w, v) \notin[0,3] \times[0,3],\end{cases}
$$

for all $(w, v) \in A \times A, f(t)=\ln (t)$, and $\mu=\ln (3)$. Moreover, $D$ does not form a metric but it is an $\mathscr{F}$-MS. 
Jleli and Samet proposed a simple Banach fixed-point theorem as follows.

Theorem 1 (see [5]). Let $(A, D)$ be an $\mathscr{F}-M S$. Let $g: A \longrightarrow A$ be a self-mapping. Suppose that the following conditions are met:

(i) $(A, D)$ is $\mathscr{F}$-complete.

(ii) $\exists$ a constant $k \in(0,1)$ such that

$$
D(g(w), g(v)) \leq k D(w, v), \quad(w, v) \in A \times A .
$$

Then, $g$ attains a unique fixed-point $w^{*} \in A$.

In 2012, Samet et al. introduced a class of $\alpha$-admissible mappings as follows.

Definition 3 (see [6]). Let $T: A \longrightarrow A$ and $\alpha: A \times A \longrightarrow$ $[0,+\infty) . T$ is said to be $\alpha$-admissible if $w, v \in A$, and $\alpha(w, v) \geq 1$ implies that $\alpha(T w, T v) \geq 1$.

Next, Salimi et al. [7] modified the concept of $\alpha$-admissible mapping as follows.

Definition 4 (see [7]). Let $T: A \longrightarrow A$ and $\alpha, \eta: A \times A \longrightarrow$ $[0,+\infty)$ be two functions. $T$ is called an $\alpha$-admissible mapping with respect to $\eta$, if $w, v \in A$, and $\alpha(w, v) \geq \eta(w, v)$ implies that $\alpha(T w, T v) \geq \eta(T w, T v)$.

If $\eta(w, v)=1$, then the above definition reduces to Definition 3. If $\alpha(w, v)=1$, then $T$ is called an $\eta$-subadmissible mapping.
Definition 5 (see [8]). Consider a metric space $(A, d)$ and assume that $T: A \longrightarrow A$ and $\alpha, \eta: A \times A \longrightarrow[0, \infty)$ are two functions. A mapping $T$ is considered as $\alpha-\eta$-continuous mapping in $(A, d)$ whenever $w \in A$ is given, and the sequence $\left\{w_{n}\right\}$ is as follows:

$$
\begin{aligned}
& w_{n} \longrightarrow w \text { at } \infty, \\
& \alpha\left(w_{n}, w_{n+1}\right) \geq \eta\left(w_{n}, w_{n+1}\right), \\
& \forall n \in \mathbb{N} \text { implies } T w_{n} \longrightarrow T w .
\end{aligned}
$$

For more details, see, for example, $[9,10]$.

A mapping $T: A \longrightarrow A$ is called orbitally continuous in $v \in A$ if $\lim _{n \longrightarrow \infty} T^{n} w=v$ implies that $\lim _{n \longrightarrow \infty} T^{n} w=T v . T$ mapping is orbitally continuous on $A$ if $T$ is orbitally continuous $\forall v \in A$.

\section{Fractional Symmetric $\alpha-\eta$-Contraction of Type-I}

In this segment, first we present a new fractional symmetric $\alpha-\eta$-contraction of type-I.

Definition 6. Let $T: A \longrightarrow A$ be an $\mathscr{F}$-metric space $(A, D)$ and two functions $\alpha, \eta: A \times A \longrightarrow[0,+\infty)$. We consider that $T$ is a fractional symmetric $\alpha-\eta$-contraction of type-I along with constants $\lambda \in[0,1)$ and $\beta, \widehat{w}, \gamma \in(0,1)$ such that, whenever $\alpha(w, v) \geq \eta(w, v)$, we have

$$
D(T w, T v)^{p} \leq \lambda\left(\check{S}_{1}(w, v)\right),
$$

where

$$
\begin{aligned}
\check{S}_{1}(w, v)= & D(w, v)^{p} \cdot[D(w, T w)]^{(p /(\beta-\widehat{w})(\beta-\gamma))}, \\
& {[D(v, T v)]^{(p /(\beta-\widehat{w})(\beta-\gamma))} \cdot[D(w, T w)+D(v, T v)]^{(p /(\widehat{w}-\beta)(\widehat{w}-\gamma))} . } \\
& {[D(w, T v)+D(v, T w)]^{(p /(\gamma-\beta)(\gamma-\widehat{w}))}, }
\end{aligned}
$$

where $p \in[1, \infty)$, for all $w, v \in A \backslash \operatorname{Fix}(T)$.

Example 2. Let $A=\{0,1,2,3\}$ with grace of $\mathscr{F}$-metric $D$ defined by

$$
D(w, v)=\left\{\begin{array}{cc}
(w-v)^{2}, & \text { if }(w, v) \in A \times A, \\
|w-v|, & \text { if }(w, v) \notin A \times A,
\end{array}\right.
$$

and consider $f(t)=\ln (t)$ and $\mu=\ln (3)$. Define $T: A \longrightarrow A$ by

$$
T 0=0, T 1=1, T 2=T 3=0,
$$

and $\alpha, \eta: A \times A \longrightarrow[0,+\infty)$ by

$$
\begin{aligned}
& \alpha(w, v)= \begin{cases}1, & \text { if } w, v \in A, \\
0, & \text { otherwise, }\end{cases} \\
& \eta(w, v)= \begin{cases}\frac{1}{2}, & \text { if } w, v \in A, \\
0, & \text { otherwise. }\end{cases}
\end{aligned}
$$




$$
\begin{aligned}
D(T 2, T 3)^{p}= & 0 \leq \lambda\left[D(2,3)^{p} \cdot D(2, T 2)^{(p /(\beta-\widehat{w})(\beta-\gamma))} \cdot D(3, T 3)^{(p /(\beta-\widehat{w})(\beta-\gamma))}\right. \\
& \left.(D(2, T 2)+D(3, T 3))^{(p /(\widehat{w}-\beta)(\widehat{w}-\gamma))}(D(2, T 3)+D(3, T 2))^{(p /(\gamma-\beta)(\gamma-\widehat{w}))}\right] \\
= & \lambda\left[1 \cdot D(2,0)^{(p /(\beta-\widehat{w})(\beta-\gamma))} D(3,0)^{(p /(\beta-\widehat{w})(\beta-\gamma))}(D(2,0)+D(3,0))^{(p /(\widehat{w}-\beta)(\widehat{w}-\gamma))}\right. \\
& \left.\cdot(D(2,0)+D(3,0))^{(p /(\gamma-\beta)(\gamma-\widehat{w}))}\right] \\
= & \lambda\left[(4)^{(p /(\beta-\widehat{w})(\beta-\gamma))} \cdot(9)^{(p /(\beta-\widehat{w})(\beta-\gamma))} \cdot(4+9)^{(p /(\widehat{w}-\beta)(\widehat{w}-\gamma))} \cdot(4+9)^{(p /(\gamma-\beta)(\gamma-\widehat{w}))}\right] \\
\leq & \lambda\left[(4)^{(p /(\beta-\widehat{w})(\beta-\gamma))} \cdot(9)^{(p /(\beta-\widehat{w})(\beta-\gamma))} \cdot(4 \cdot 9)^{(p /(\widehat{w}-\beta)(\widehat{w}-\gamma))} \cdot(4 \cdot 9)^{(p /(\gamma-\beta)(\gamma-\widehat{w}))}\right] \\
= & \lambda[(4) \cdot(9)]^{(p /(\beta-\widehat{w})(\beta-\gamma))+(p /(\widehat{w}-\beta)(\widehat{w}-\gamma))+(p /(\gamma-\beta)(\gamma-\widehat{w}))}=\lambda .
\end{aligned}
$$

By taking any value of constants $\lambda \in[0,1)$ and $\beta, \widehat{w}, \gamma \in(0,1)$, clearly, (6) holds for all $p \in[1, \infty)$, $w, v \in A \backslash \operatorname{Fix}(T)$. Point out that $T$ has two fixed points, which are 0 and 1.

Now, we initiate brand new fixed-point theorems for fractional symmetric $\alpha-\eta$-contraction of type-I in the configuration of $\mathscr{F}$-complete $\mathscr{F}$-MS.

Theorem 2. Let $(A, D)$ be a complete $\mathscr{F}$-metric space and $T$ is a fractional symmetric $\alpha-\eta$-contraction of type-I satisfying the following statements:

(i) $T$ is an $\alpha$-admissible mapping concerning $\eta$

(ii) There exists $w_{0} \in A$ to such an extent that $\alpha\left(w_{0}, T w_{0}\right) \geq \eta\left(w_{0}, T w_{0}\right)$

(iii) $T$ is $\alpha$ - $\eta$-continuous

At that point, $T$ possesses a fixed point at $A$.
Proof. Consider $w_{0}$ in $A$ with the goal that $\alpha\left(w_{0}, T w_{0}\right) \geq$ $\eta\left(w_{0}, T w_{0}\right)$. For $w_{0} \in A$, we build a chain $\left\{w_{n}\right\}_{n=1}^{\infty}$ in such a way that $w_{1}=T w_{0}$ and $w_{2}=T w_{1}=T^{2} w_{0}$. Proceeding with this exercise, $w_{n+1}=T w_{n}=T^{n+1} w_{0}$, for every $n \in \mathbb{N}$. Presently, as long as mapping $T$ is $\alpha$-admissible with respect to $\eta$, at that time $\alpha\left(w_{0}, w_{1}\right)=\alpha\left(w_{0}, T w_{0}\right)$ $\geq \eta\left(w_{0}, T w_{0}\right)=\eta\left(w_{0}, w_{1}\right)$. Carrying on in this way, we get $\alpha\left(w_{n-1}, w_{n}\right) \geq \eta\left(w_{n-1}, w_{n}\right)=\eta\left(w_{n-1}, T w_{n-1}\right), \quad$ for all $n \in \mathbb{N}$.

Provided that $w_{n+1}=w_{n}$ for some $n \in \mathbb{N}$, then $w_{n}=w^{*}$ is a fixed point of $T$. So, we assume that $w_{n} \neq w_{n+1}$ accompanied by

$$
D\left(T w_{n-1}, T w_{n}\right)=D\left(w_{n}, T w_{n}\right)>0, \quad \text { for all } n \in \mathbb{N} .
$$

As $T$ is fractional symmetric $\alpha-\eta$-contraction of type-I, a part of $n \in \mathbb{N}$, we have

$$
\begin{aligned}
D\left(w_{n}, w_{n+1}\right)^{p}= & D\left(T w_{n-1}, T w_{n}\right)^{p} \leq \lambda\left[D\left(w_{n-1}, w_{n}\right)^{p} \cdot D\left(w_{n-1}, T w_{n-1}\right)^{(p /(\beta-\widehat{w})(\beta-\gamma))} \cdot D\left(w_{n}, T w_{n}\right)^{(p /(\beta-\widehat{w})(\beta-\gamma))} .\right. \\
& {\left.\left[D\left(w_{n-1}, T w_{n-1}\right)+D\left(w_{n}, T w_{n}\right)\right]^{(p /(\widehat{w}-\beta)(\widehat{w}-\gamma))} \cdot\left[D\left(w_{n-1}, T w_{n}\right)+D\left(w_{n}, T w_{n-1}\right)\right]^{(p /(\gamma-\beta)(\gamma-\widehat{w}))}\right] } \\
= & \lambda\left[D\left(w_{n-1}, w_{n}\right)^{p} \cdot D\left(w_{n-1}, w_{n}\right)^{(p /(\beta-\widehat{w})(\beta-\gamma))} \cdot D\left(w_{n}, w_{n+1}\right)^{(p /(\beta-\widehat{w})(\beta-\gamma))} \cdot\right. \\
& {\left.\left[D\left(w_{n-1}, w_{n}\right)+D\left(w_{n}, w_{n+1}\right)\right]^{(p /(\widehat{w}-\beta)(\widehat{w}-\gamma))} \cdot\left[D\left(w_{n-1}, w_{n+1}\right)+D\left(w_{n}, w_{n}\right)\right]^{(p /(\gamma-\beta)(\gamma-\widehat{w}))}\right] } \\
\leq & \lambda\left[D\left(w_{n-1}, w_{n}\right)^{p} \cdot D\left(w_{n-1}, w_{n}\right)^{(p /(\beta-\widehat{w})(\beta-\gamma))} \cdot D\left(w_{n}, w_{n+1}\right)^{(p /(\beta-\widehat{w})(\beta-\gamma))} .\right. \\
& {\left.\left[D\left(w_{n-1}, w_{n}\right)+D\left(w_{n}, w_{n+1}\right)\right]^{(p /(\widehat{w}-\beta)(\widehat{w}-\gamma))} \cdot\left[D\left(w_{n-1}, w_{n}\right)+D\left(w_{n}, w_{n+1}\right)\right]^{(p /(\gamma-\beta)(\gamma-\widehat{w}))}\right] } \\
= & \lambda\left[D\left(w_{n-1}, w_{n}\right)^{p} \cdot D\left(w_{n-1}, w_{n}\right)^{(p /(\beta-\widehat{w})(\beta-\gamma))} \cdot D\left(w_{n}, w_{n+1}\right)^{(p /(\beta-\widehat{w})(\beta-\gamma))} .\right. \\
& {\left.\left[D\left(w_{n-1}, w_{n}\right)+D\left(w_{n}, w_{n+1}\right)\right]^{(p /(\widehat{w}-\beta)(\widehat{w}-\gamma))+(p /(\gamma-\beta)(\gamma-\widehat{w}))}\right] } \\
\leq & \lambda\left[D\left(w_{n-1}, w_{n}\right)^{p} \cdot D\left(w_{n-1}, w_{n}\right)^{(p /(\beta-\widehat{w})(\beta-\gamma))} \cdot D\left(w_{n}, w_{n+1}\right)^{(p /(\beta-\widehat{w})(\beta-\gamma))} .\right. \\
& {\left.\left[D\left(w_{n-1}, w_{n}\right) \cdot D\left(w_{n}, w_{n+1}\right)\right]^{(p /(\widehat{w}-\beta)(\widehat{w}-\gamma))+(p /(\gamma-\beta)(\gamma-\widehat{w}))}\right] } \\
= & \lambda\left[D\left(w_{n-1}, w_{n}\right)^{p+(p /(\beta-\widehat{w})(\beta-\gamma))+(p /(\widehat{w}-\beta)(\widehat{w}-\gamma))+(p /(\gamma-\beta)(\gamma-\widehat{w}))}\right] . \\
& D\left(w_{n}, w_{n+1}\right)^{(p /(\beta-\widehat{w})(\beta-\gamma))+(p /(\widehat{w}-\beta)(\widehat{w}-\gamma))+(p /(\gamma-\beta)(\gamma-\widehat{w}))} \\
= & \lambda D\left(w_{n-1}, w_{n}\right)^{p},
\end{aligned}
$$


which implies that

$$
D\left(w_{n}, w_{n+1}\right)^{p} \leq \lambda D\left(w_{n-1}, w_{n}\right)^{p},
$$

and we deduce that

$$
D\left(w_{n}, w_{n+1}\right) \leq \lambda D\left(w_{n-1}, w_{n}\right) .
$$

We conclude that $\left\{D\left(w_{n-1}, w_{n}\right)\right\}$ is a nonincreasing sequence with nonnegative terms. Thus, there is a nonnegative constant $\varrho$ such that $\lim _{n \longrightarrow \infty} D\left(w_{n-1}, w_{n}\right)=\varrho$. Note that $\varrho \geq 0$. From (16), we have

$$
D\left(w_{n}, w_{n+1}\right) \leq \lambda D\left(w_{n-1}, w_{n}\right) \leq \lambda^{n} D\left(w_{0}, w_{1}\right) .
$$

This provides

$$
\sum_{i=n}^{m-1} D\left(w_{i}, w_{i+1}\right) \leq \frac{\lambda^{n}}{1-\lambda} D\left(w_{0}, w_{1}\right), \quad m>n .
$$

Considering for as much as

$$
\lim _{n \longrightarrow+\infty} \frac{\lambda^{n}}{1-\lambda} D\left(w_{0}, w_{1}\right)=0,
$$

there subsist some $N \in \mathbb{N}$ corresponding to

$$
0<\frac{\lambda^{n}}{1-\lambda} D\left(w_{0}, w_{1}\right)<\delta, \quad n \geq N .
$$

Let $\varepsilon>0$ be fixed and let $(f, \mu) \in \mathscr{F} \times[0, \infty)$ be cognate and $\left(D_{3}\right)$ is satisfied. By $\left(\mathscr{F}_{2}\right)$, there exists $\delta>0$ which connotes that

$$
0<t<\delta \text { implies } f(t)<f(\varepsilon)-\mu .
$$

Hence, by $(21)$ and $\left(\mathscr{F}_{1}\right)$, we get

$$
f\left(\sum_{i=n}^{m-1} D\left(w_{i}, w_{i+1}\right)\right) \leq f\left(\frac{\lambda^{n}}{1-\lambda} D\left(w_{0}, w_{1}\right)\right)<f(\varepsilon)-\mu
$$

where $m, n \in \mathbb{N}$ with the goal that $m>n \geq N$ together with $D\left(w_{n}, w_{m}\right)>0$. Therefore, by using $\left(D_{3}\right)$ and $(22)$, we have

$$
f\left(D\left(w_{m}, w_{n}\right)\right) \leq f\left(\sum_{i=n}^{m-1}\left(D\left(w_{i}, w_{i+1}\right)\right)\right)+\mu<f(\varepsilon),
$$

which implies that by $\left(\mathscr{F}_{1}\right)$, we have

$$
D\left(w_{m}, w_{n}\right)<\varepsilon, \quad \text { for } m>n \geq N .
$$

Consequently, $\left\{w_{n}\right\}$ is an $\mathscr{F}$-Cauchy sequence. Meanwhile $(A, D)$ is an $\mathscr{F}$-complete metric space and there exists $w^{*} \in A$ such that $w_{n}$ is $\mathscr{F}$-convergent to $w^{*}$; that is,

$$
\lim _{n \longrightarrow \infty} D\left(w_{n}, w^{*}\right)=0 \text {, }
$$

and $T$ is $\alpha-\eta$-continuous as well as $\alpha\left(w_{n-1}, w_{n}\right) \geq \eta\left(w_{n-1}\right.$, $\left.w_{n}\right)$, each one of $n \in \mathbb{N}$ at that point $w_{n+1}=T w_{n} \longrightarrow T w^{*}$ as $n \longrightarrow \infty$; in other words, $w^{*}=T w^{*}$. Now we are going to prove that $w^{*}$ is a fixed point of $T$. We argue by contradiction by supposing that $D\left(T w^{*}, w^{*}\right)>0$. By $(D 3)$, we have

$$
\begin{aligned}
f\left(D\left(T w^{*}, w^{*}\right)^{p}\right) \leq & f\left(D\left(T w^{*}, T w_{n}\right)^{p}+D\left(T w_{n}, w^{*}\right)^{p}\right) \\
& +\mu, \quad n \in \mathbb{N} .
\end{aligned}
$$

By using $\left(\mathscr{F}_{1}\right)$ and the contractive condition, we have

$$
\begin{aligned}
f\left(D\left(T w^{*}, w^{*}\right)\right) \leq & f\left(\lambda \left(D\left(w^{*}, w_{n}\right)^{p} \cdot D\left(T w^{*}, w^{*}\right)^{(p /(\beta-\widehat{w})(\beta-\gamma))} \cdot D\left(w_{n}, T w_{n}\right)^{(p /(\beta-\widehat{w})(\beta-\gamma))}\right.\right. \\
\cdot & {\left[D\left(T w^{*}, w^{*}\right)+D\left(w_{n}, T w_{n}\right)\right]^{(p /(\widehat{w}-\beta)(\widehat{w}-\gamma))} } \\
\cdot & {\left.\left.\left[D\left(T w_{n}, w^{*}\right)+D\left(w_{n}, T w^{*}\right)\right]^{(p /(\gamma-\beta)(\gamma-\widehat{w}))}+D\left(w_{n+1}, w^{*}\right)^{p}\right)\right)+\mu }
\end{aligned}
$$

for all $n \in \mathbb{N}$. In other words, by using $\left(\mathscr{F}_{2}\right)$ and $(25)$, we get

$$
\lim _{n \rightarrow \infty} f\left(\lambda D\left(w^{*}, w_{n}\right)^{p}+D\left(w_{n+1}, w^{*}\right)^{p}\right)+\mu=-\infty,
$$

which gives a contradiction. Therefore $D\left(T w^{*}, w^{*}\right)=0$; hence $w^{*}$ possesses a fixed point of $T$.

Theorem 3. Let $(A, D)$ be an $\mathscr{F}$-complete $\mathscr{F}$-metric space and let $T$ be a fractional symmetric $\alpha-\eta$-contraction of type-I fulfilling the accompanying affirmations:

(i) $T$ is an $\alpha$-admissible mapping concerning $\eta$

(ii) There exists a $w_{0} \in A$ to such extent that $\alpha\left(w_{0}, T w_{0}\right) \geq \eta\left(w_{0}, T w_{0}\right)$ (iii) An iteration $\left\{w_{n}\right\}$ in $A$ is such that $\alpha\left(w_{n}, w_{n+1}\right) \geq$ $\eta\left(w_{n}, w_{n+1}\right)$ escorted by $w_{n} \longrightarrow w^{*}$ at the same time $n \longrightarrow \infty$; after that $\alpha\left(w_{n}, w^{*}\right) \geq \eta\left(w_{n}, w^{*}\right)$ holds for each $n \in \mathbb{N}$

Afterwards, $T$ possesses a fixed point in A.

Proof. On closing lines of the proof of Theorem 2, we acquire $\alpha\left(w_{n}, w^{*}\right) \geq \eta\left(w_{n}, w^{*}\right)$ for each $n \in \mathbb{N}$. Using $\left(D_{3}\right)$, we have

$$
f\left(D\left(T w^{*}, w^{*}\right)^{p}\right) \leq f\left(D\left(T w^{*}, T w_{n}\right)^{p}+D\left(w_{n}, w^{*}\right)^{p}\right)+\mu .
$$

From (6) connecting $\left(\mathscr{F}_{1}\right)$, we have 


$$
\begin{aligned}
f\left(D\left(T w^{*}, w^{*}\right)^{p}\right) \leq & f\left(\left(D\left(T w^{*}, T w_{n}\right)^{p}\right)+D\left(T w_{n}, w^{*}\right)^{p}\right)+\mu \\
\leq & f\left(\lambda \left(D\left(w^{*}, w_{n}\right)^{p} \cdot D\left(T w^{*}, w^{*}\right)^{(p /(\beta-\widehat{w})(\beta-\gamma))} \cdot D\left(w_{n}, T w_{n}\right)^{(p /(\beta-\widehat{w})(\beta-\gamma))}\right.\right. \\
\cdot & {\left[D\left(T w^{*}, w^{*}\right)+D\left(w_{n}, T w_{n}\right)\right]^{(p /(\widehat{w}-\beta)(\widehat{w}-\gamma))} } \\
& \left.\left.\cdot\left[D\left(T w_{n}, w^{*}\right)+D\left(w_{n}, T w^{*}\right)\right]^{(p /(\gamma-\beta)(\gamma-\widehat{w}))}+D\left(w_{n+1}, w^{*}\right)^{p}\right)\right)+\mu .
\end{aligned}
$$

Employ (25) with certitude that

$\lim _{n \longrightarrow \infty} D\left(w_{n}, w^{*}\right)=0, \quad$ together with $\lim _{n \longrightarrow \infty} D\left(w_{n+1}, w^{*}\right)=0$,

and we obtain

$$
f\left(D\left(w^{*}, T w^{*}\right)^{p}\right) \leq f\left(D\left(w^{*}, T w^{*}\right)^{p}\right)+\mu,
$$

and making use of $\left(\mathscr{F}_{2}\right)$, we have

$$
\lim _{n \longrightarrow \infty} f\left(D\left(w^{*}, T w^{*}\right)^{p}\right)+\mu=-\infty
$$

which is a contradiction. Therefore, $D\left(w^{*}, T w^{*}\right)=0$; in other words, $w^{*}$ possesses a fixed point of $T$.

Example 3. Let $A=(0,1] \subset \mathbb{R}$ with an $\mathscr{F}$-metric $D: A \times$ $A \longrightarrow[0, \infty)$ by

$$
D(w, v)= \begin{cases}(w-v)^{2}, & \text { if }(w, v) \in A \times A, \\ |w-v|, & \text { if }(w, v) \notin A \times A,\end{cases}
$$

accompanied by $f(t)=\ln (t)$ together with $\mu=\ln (1), p=1$. Define $T: A \longrightarrow A$ along with

$$
T w= \begin{cases}\frac{w^{2}}{e^{6}}, & \text { if } w \in A, \\ 0, & \text { if } w \notin A,\end{cases}
$$

and $\alpha, \eta: A \times A \longrightarrow[0,+\infty)$ by

$$
\begin{aligned}
& \alpha(w, v)= \begin{cases}2, & \text { if } w, v \in[0, \infty), \\
0, & \text { otherwise }\end{cases} \\
& \eta(w, v)= \begin{cases}1, & \text { if } w, v \in[0, \infty), \\
0, & \text { otherwise. }\end{cases}
\end{aligned}
$$

(i) Case I. If $w=v$, clearly $D(w, v)=0$. Hence, every condition of Theorem 2 is satisfied.

(ii) Case II. If $w, v \in A$, clearly $T$ is an $\alpha$-admissible mapping with respect to $\eta$, whenever $\alpha(w, v) \geq \eta(w, v)$, such that

$$
\begin{aligned}
D(T w, T v)= & \frac{1}{e^{6}}\left|w^{2}-v^{2}\right| \leq \lambda\left[(v-w)^{2} \cdot\left(w-\frac{w}{e}\right)^{(1 /(\beta-\widehat{w})(\beta-\gamma))} \cdot\left(v-\frac{v}{e}\right)^{(1 /(\beta-\widehat{w})(\beta-\gamma))} .\right. \\
& \left.\left(\left(w-\frac{w}{e}\right)+\left(v-\frac{v}{e}\right)\right)^{(1 /(\widehat{w}-\beta)(\widehat{w}-\gamma))} \cdot\left(\left|w-\frac{v}{e}\right|+\left|v-\frac{w}{e}\right|\right)^{(1 /(\gamma-\beta)(\gamma-\widehat{w}))}\right]
\end{aligned}
$$

(iii) By taking constant $\lambda \in[0,1)$, and $\beta, \widehat{w}, \gamma \in(0,1)$,

for all $w, v \in A \backslash \operatorname{Fix}(T)$.

(iv) Case III. If any $w, v \notin A$, then we have

$$
\begin{aligned}
D(T w, T v)= & 0 \leq \lambda\left[|v-w| \cdot|w|^{(1 /(\beta-\widehat{w})(\beta-\gamma))} \cdot|v|^{(1 /(\beta-\widehat{w})(\beta-\gamma))}\right. \\
& \left.\cdot(|w|+|v|)^{(1 /(\widehat{w}-\beta)(\widehat{w}-\gamma))} \cdot(|w|+|v|)^{(1 /(\gamma-\beta)(\gamma-\widehat{w}))}\right] .
\end{aligned}
$$

Therefore, whole constraints of Theorem 2 are satisfied. Hence, $T$ is fractional symmetric $\alpha-\eta$-contraction of type-I.

Definition 7. Consider an $\mathscr{F}$-metric space $(A, D)$ and two functions $\alpha, \eta: A \times A \longrightarrow[0,+\infty)$. Then an $\mathscr{F}$-metric space on $A$ is said to be $\alpha-\eta$-complete if and only if every $\mathscr{F}$-Cauchy sequence $\left\{w_{n}\right\}$, along with

$$
\alpha\left(w_{n}, w_{n+1}\right) \geq \eta\left(w_{n}, w_{n+1}\right) \text { each one of the } n \in \mathbb{N} .
$$

$\mathscr{F}$-converges in $A$. 
Remark 1. Theorems 2 and 3 also hold for $\alpha$ - $\eta$-complete $\mathscr{F}$ -metric space instead of $\mathscr{F}$-complete $\mathscr{F}$-metric space (for details, see [10]).

\section{Fractional Symmetric $\alpha-\eta$-Contraction of Type-II}

In this section, a fractional symmetric $\alpha-\eta$-contraction of type-II is introduced and in the structure of $\mathscr{F}$-complete $\mathscr{F}$-metric space. Using this notion, we shall provide a fixedpoint theorem.
Definition 8. Consider a self-map $T: A \longrightarrow A$ on an $\mathscr{F}$-metric space $(A, D)$ and two functions $\alpha, \eta: A \times A \longrightarrow[0,+\infty)$. We presume that $T$ is a fractional symmetric $\alpha-\eta$-contraction of type-II provided that there are constants $\lambda \in[0,1)$ and $\beta, \widehat{w}, \gamma \in(0,1)$ such that, whenever $\alpha(w, v) \geq \eta(w, v)$, we own

$$
D(T w, T v)^{p} \leq \lambda\left(\check{S}_{2}(w, v)\right),
$$

where

$$
\begin{aligned}
\check{S}_{2}(w, v)= & \left\{D(w, v) \cdot[D(w, T w)]^{(p \beta /(\beta-\widehat{w})(\beta-\gamma))} \cdot[D(v, T v)]^{(p \beta /(\beta-\widehat{w})(\beta-\gamma))} \cdot\right. \\
& {\left.[D(w, T w)+D(v, T v)]^{(p \widehat{w}(\widehat{w}-\beta)(\widehat{w}-\gamma))} \cdot[D(w, T v)+D(v, T w)]^{(p \gamma /(\gamma-\beta)(\gamma-\widehat{w}))}\right\}, }
\end{aligned}
$$

where $p \in[1, \infty)$, for all $w, v \in A \backslash \operatorname{Fix}(T)$.

Now we show and demonstrate our next theorem.

Theorem 4. Let $(A, D)$ be an $\mathscr{F}$-complete $\mathscr{F}$-metric space and let $T$ be a fractional symmetric $\alpha-\eta$-contraction of type-II fulfilling the accompanying affirmations:

(i) $T$ is an $\alpha$-admissible mapping concerning $\eta$

(ii) There exists $w_{0} \in A$ to such an extent that $\alpha\left(w_{0}, T w_{0}\right) \geq \eta\left(w_{0}, T w_{0}\right)$

(iii) $T$ is $\alpha$ - $\eta$-continuous

After that, $T$ possesses a fixed point in A.

Proof. Consider $w_{0}$ in $A$ correspondent to $\alpha\left(w_{0}, T w_{0}\right) \geq$ $\eta\left(w_{0}, T w_{0}\right)$. For $w_{0} \in A$, we build an iteration $\left\{w_{n}\right\}_{n=1}^{\infty}$ in such a way that $w_{1}=T w_{0}$ and $w_{2}=T w_{1}=T^{2} w_{0}$.
Proceeding with this exercise, $w_{n+1}=T w_{n}=T^{n+1} w_{0}$, for all $n \in \mathbb{N}$. Now, as long as mapping $T$ is $\alpha$-admissible with respect to $\eta$, at that time $\alpha\left(w_{0}, w_{1}\right)=\alpha\left(w_{0}, T w_{0}\right) \geq \eta\left(w_{0}\right.$, $\left.T w_{0}\right)=\eta\left(w_{0}, w_{1}\right)$. Carrying on in this way, we own

$\alpha\left(w_{n-1}, w_{n}\right) \geq \eta\left(w_{n-1}, w_{n}\right)=\eta\left(w_{n-1}, T w_{n-1}\right), \quad$ for all $n \in \mathbb{N}$.

Provided that $w_{n+1}=w_{n}$ for some $n \in \mathbb{N}$, then $w_{n}=w^{*}$ is a fixed point of $T$. So, we assume that $w_{n} \neq w_{n+1}$ accompanied by

$$
D\left(T w_{n-1}, T w_{n}\right)=D\left(w_{n}, T w_{n}\right)>0, \quad \text { every } n \in \mathbb{N} .
$$

As $T$ is fractional symmetric $\alpha-\eta$-contraction of type-II, a part of $n \in \mathbb{N}$, we own

$$
\begin{aligned}
& D\left(w_{n}, w_{n+1}\right)^{p}=D\left(T w_{n-1}, T w_{n}\right)^{p} \leq \lambda\left[D\left(w_{n-1}, w_{n}\right)^{p} \cdot D\left(w_{n-1}, T w_{n-1}\right)^{(p \beta /(\beta-\widehat{w})(\beta-\gamma))} \cdot D\left(w_{n}, T w_{n}\right)^{(p \beta /(\beta-\widehat{w})(\beta-\gamma))} .\right. \\
& \left.\left[D\left(w_{n-1}, T w_{n-1}\right)+D\left(w_{n}, T w_{n}\right)\right]^{(p \widehat{w}(\widehat{w}-\beta)(\widehat{w}-\gamma))} \cdot\left[D\left(w_{n-1}, T w_{n}\right)+D\left(w_{n}, T w_{n-1}\right)\right]^{(p \gamma /(\gamma-\beta)(\gamma-\widehat{w}))}\right] \\
& =\lambda\left[D\left(w_{n-1}, w_{n}\right)^{p} \cdot D\left(w_{n-1}, w_{n}\right)^{(p \beta /(\beta-\widehat{w})(\beta-\gamma))} \cdot D\left(w_{n}, w_{n+1}\right)^{(p \beta /(\beta-\widehat{w})(\beta-\gamma))}\right. \text {. } \\
& \left.\left[D\left(w_{n-1}, w_{n}\right)+D\left(w_{n}, w_{n+1}\right)\right]^{(p w /(\widehat{w}-\beta)(\widehat{w}-\gamma))} \cdot\left[D\left(w_{n-1}, w_{n+1}\right)+D\left(w_{n}, w_{n}\right)\right]^{(p \gamma /(\gamma-\beta)(\gamma-\widehat{w}))}\right] \\
& \leq \lambda\left[D\left(w_{n-1}, w_{n}\right)^{p} \cdot D\left(w_{n-1}, w_{n}\right)^{(p \beta /(\beta-\widehat{w})(\beta-\gamma))} \cdot D\left(w_{n}, w_{n+1}\right)^{(p \beta /(\beta-\widehat{w})(\beta-\gamma))}\right. \text {. } \\
& \left.\left[D\left(w_{n-1}, w_{n}\right)+D\left(w_{n}, w_{n+1}\right)\right]^{(p \hat{w /}(\widehat{w}-\beta)(\widehat{w}-\gamma))} \cdot\left[D\left(w_{n-1}, w_{n}\right)+D\left(w_{n}, w_{n+1}\right)\right]^{(p \gamma /(\gamma-\beta)(\gamma-\widehat{w}))}\right] \\
& =\lambda\left[D\left(w_{n-1}, w_{n}\right)^{p} \cdot D\left(w_{n-1}, w_{n}\right)^{(p \beta /(\beta-\widehat{w})(\beta-\gamma))} \cdot D\left(w_{n}, w_{n+1}\right)^{(p \beta /(\beta-\widehat{w})(\beta-\gamma))}\right. \text {. } \\
& \left.\left[D\left(w_{n-1}, w_{n}\right)+D\left(w_{n}, w_{n+1}\right)\right]^{(p \widehat{w}(\widehat{w}-\beta)(\widehat{w}-\gamma))+(p \gamma /(\gamma-\beta)(\gamma-\widehat{w}))}\right] \\
& \leq \lambda\left[D\left(w_{n-1}, w_{n}\right)^{p} \cdot D\left(w_{n-1}, w_{n}\right)^{(p \beta /(\beta-\widehat{w})(\beta-\gamma))} \cdot D\left(w_{n}, w_{n+1}\right)^{(p \beta /(\beta-\widehat{w})(\beta-\gamma))} .\right. \\
& \left.\left[D\left(w_{n-1}, w_{n}\right) \cdot D\left(w_{n}, w_{n+1}\right)\right]^{(p \widehat{w /}(\widehat{w}-\beta)(\widehat{w}-\gamma))+(p \gamma /(\gamma-\beta)(\gamma-\widehat{w}))}\right] \\
& =\lambda\left[D\left(w_{n-1}, w_{n}\right)^{p+(p \beta /(\beta-\widehat{w})(\beta-\gamma))+(p \widehat{w} /(\widehat{w}-\beta)(\widehat{w}-\gamma))+(p \gamma /(\gamma-\beta)(\gamma-\widehat{w}))}\right] \text {. } \\
& D\left(w_{n}, w_{n+1}\right)^{(p \beta /(\beta-\widehat{w})(\beta-\gamma))+(p \widehat{w}(\widehat{w}-\beta)(\widehat{w}-\gamma))+(p \gamma /(\gamma-\beta)(\gamma-\widehat{w}))} \\
& =\lambda D\left(w_{n-1}, w_{n}\right)^{p} \text {, }
\end{aligned}
$$


which implies that

$$
D\left(w_{n}, w_{n+1}\right)^{p} \leq \lambda D\left(w_{n-1}, w_{n}\right)^{p},
$$

and we deduce that

$$
D\left(w_{n}, w_{n+1}\right) \leq \lambda D\left(w_{n-1}, w_{n}\right) .
$$

We conclude that $\left\{D\left(w_{n-1}, w_{n}\right)\right\}$ is a nonincreasing sequence with nonnegative terms. As a result, there is a nonnegative constant $\rho$ such that $\lim _{n \rightarrow \infty} D\left(w_{n-1}, w_{n}\right)=\rho$. We shall indicate that $\rho>0$. Indeed, from (46), we derive that

$$
D\left(w_{n}, w_{n+1}\right) \leq \lambda D\left(w_{n-1}, w\right) \leq \lambda^{n} D\left(w_{0}, w_{1}\right) .
$$
2

The rest of the test follows the same lines of Theorem

Theorem 5. Consider an $\mathscr{F}$-complete $\mathscr{F}$-metric space $(A, D)$ and let $T$ be a fractional symmetric $\alpha-\eta$-contraction of type-II meeting the following assertions: (i) $T$ is an $\alpha$-admissible mapping with respect to $\eta$

(ii) There exists $w_{0} \in A$ such that $\alpha\left(w_{0}, T w_{0}\right) \geq$ $\eta\left(w_{0}, T w_{0}\right)$

(iii) An iteration $\left\{w_{n}\right\}$ in $A$ is such that $\alpha\left(w_{n}, w_{n+1}\right) \geq$ $\eta\left(w_{n}, w_{n+1}\right)$ escorted by $w_{n} \longrightarrow w^{*}$ at the same time $n \longrightarrow \infty$; after that $\alpha\left(w_{n}, w^{*}\right) \geq \eta\left(w_{n}, w^{*}\right)$ holds for each $n \in \mathbb{N}$

Afterwards, $T$ possesses a fixed point in A.

Proof. Similar to the lines of Theorem 3, since, by (iii), $\alpha\left(w_{n}, w^{*}\right) \geq \eta\left(w_{n}, w^{*}\right)$ holds for every $n \in \mathbb{N}$. Using $\left(D_{3}\right)$, we meet

$$
f\left(D\left(T w^{*}, w^{*}\right)\right) \leq f\left(D\left(T w^{*}, T w_{n}\right)+D\left(w_{n}, w^{*}\right)\right)+\mu .
$$

From $(40)$ and $\left(\mathscr{F}_{1}\right)$, we have

$$
\begin{aligned}
f\left(D\left(T w^{*}, w^{*}\right)^{p}\right) \leq & f\left(\left(D\left(T w^{*}, T w_{n}\right)^{p}\right)+D\left(T w_{n}, w^{*}\right)^{p}\right)+\mu \\
\leq & f\left(\lambda \left(D\left(w^{*}, w_{n}\right)^{p} \cdot D\left(T w^{*}, w^{*}\right)^{(p \beta /(\beta-\widehat{w})(\beta-\gamma))} \cdot D\left(w_{n}, T w_{n}\right)^{(p \beta /(\beta-\widehat{w})(\beta-\gamma))}\right.\right. \\
& \cdot\left[D\left(T w^{*}, w^{*}\right)+D\left(w_{n}, T w_{n}\right)\right]^{(p \widehat{w} /(\widehat{w}-\beta)(\widehat{w}-\gamma))} \\
& \left.\left.\cdot\left[D\left(T w_{n}, w^{*}\right)+D\left(w_{n}, T w^{*}\right)\right]^{(p \gamma /(\gamma-\beta)(\gamma-\widehat{w}))}+D\left(w_{n+1}, w^{*}\right)^{p}\right)\right)+\mu .
\end{aligned}
$$

Making use of (25), we get

$$
\lim _{n \longrightarrow \infty} D\left(w_{n}, w^{*}\right)=0 \text { together } \lim _{n \longrightarrow \infty} D\left(w_{n+1}, w^{*}\right)=0 \text {, }
$$

and we procure

$$
f\left(D\left(w^{*}, T w^{*}\right)\right) \leq f\left(D\left(w^{*}, T w^{*}\right)\right)+\mu .
$$

Using $\left(\mathscr{F}_{2}\right)$, we have

$$
\lim _{n \longrightarrow \infty} f\left(D\left(w^{*}, T w^{*}\right)^{p}\right)+\mu=-\infty,
$$

which is a logical inconsistency. Along these lines $D\left(w^{*}, T w^{*}\right)=0$; that is, $w^{*}$ possesses a fixed point of $T$.

\section{Fractional Symmetric $\alpha-\eta$-Contraction of Type-III}

In this section, fractional symmetric $\alpha-\eta$-contraction of type-III is considered in the environment of $\mathscr{F}$-complete $\mathscr{F}$-metric space. After stating a fixed-point theorem for such maps, we set up fractional symmetric $\alpha-\eta$-contraction of type-III as follows.

Definition 9. Consider an $\mathscr{F}$-metric space $(A, D)$ with a selfmap $T: A \longrightarrow A$ and two functions $\alpha, \eta: A \times A \longrightarrow[0$, $+\infty)$. We say that $T$ is fractional symmetric $\alpha-\eta$-contraction of type-III along with constants $\lambda \in[0,1)$ and $\beta, \widehat{w}, \gamma \in(0,1)$ such that, whenever $\alpha(w, v) \geq \eta(w, v)$, we have

$$
D(T w, T v)^{p} \leq \lambda\left(\check{S}_{3}(w, v)\right),
$$

where

$$
\begin{aligned}
\check{S}_{3}(w, v)= & \lambda \max \left\{D(w, v),[D(w, T w)]{ }^{\left(p \beta^{2} /(\beta-\widehat{w})(\beta-\gamma)\right)}\right. \\
& \cdot[D(v, T v)]\left(p \beta^{2} /(\beta-\widehat{w})(\beta-\gamma)\right) \cdot[D(w, T w)+D(v, T v)]\left(p \widehat{w}^{2} /(\widehat{w}-\beta)(\widehat{w}-\gamma)\right) \\
& \left.\cdot[D(w, T v)+D(v, T w)]\left(p \gamma^{2} /(\gamma-\beta)(\gamma-\widehat{w})\right)\right\},
\end{aligned}
$$


where $p \in[1, \infty)$, for all $w, v \in A \backslash \operatorname{Fix}(T)$.

Now we declare and demonstrate our next theorem.

Theorem 6. Let an $\mathscr{F}$-complete $(A, D)$ be an $\mathscr{F}$-metric space along with $T$ being a fractional symmetric $\alpha-\eta$-contraction of type-III which meets the following assertions:

(i) $T$ is an $\alpha$-admissible mapping concerning $\eta$

(ii) There exists $w_{0} \in A$ such that $\alpha\left(w_{0}, T w_{0}\right) \geq$ $\eta\left(w_{0}, T w_{0}\right)$

(iii) $T$ is $\alpha$ - $\eta$-continuous

After that, $T$ possesses a fixed point in A.

Proof. Consider $w_{0}$ in $A$ with the aim that $\alpha\left(w_{0}, T w_{0}\right) \geq \eta\left(w_{0}, T w_{0}\right)$. Take any $w_{0} \in A$; we erect an recapitulate $\left\{w_{n}\right\}_{n=1}^{\infty}$ in such a way that $w_{1}=T w_{0}$ and
$w_{2}=T w_{1}=T^{2} w_{0}$. Continuing with this practice, $w_{n+1}=T w_{n}=T^{n+1} w_{0}$, every $n \in \mathbb{N}$. As long as mapping $T$ is $\alpha$-admissible with respect to $\eta$, at that time $\alpha\left(w_{0}, w_{1}\right)=$ $\alpha\left(w_{0}, T w_{0}\right) \geq \eta\left(w_{0}, T w_{0}\right)=\eta\left(w_{0}, w_{1}\right)$. Carrying on in this way, we find

$\alpha\left(w_{n-1}, w_{n}\right) \geq \eta\left(w_{n-1}, w_{n}\right)=\eta\left(w_{n-1}, T w_{n-1}\right), \quad$ for all $n \in \mathbb{N}$.

Provided that $w_{n+1}=w_{n}$ for some $n \in \mathbb{N}$, then $w_{n}=w^{*}$ is a fixed point of $T$. So, we assume that $w_{n} \neq w_{n+1}$ accompanied by

$$
D\left(T w_{n-1}, T w_{n}\right)=D\left(w_{n}, T w_{n}\right)>0, \quad \text { each } n \in \mathbb{N} .
$$

As $T$ is fractional symmetric $\alpha-\eta$-contraction of type-III, a part of $n \in \mathbb{N}$, we own

$$
\begin{aligned}
& D\left(w_{n}, w_{n+1}\right)^{p}=D\left(T w_{n-1}, T w_{n}\right)^{p} \leq \lambda \max \left[D\left(w_{n-1}, w_{n}\right)^{p} \cdot D\left(w_{n-1}, T w_{n-1}\right)^{\left(p \beta^{2} /(\beta-\widehat{w})(\beta-\gamma)\right)} \cdot D\left(w_{n}, T w_{n}\right)^{\left(p \beta^{2} /(\beta-\widehat{w})(\beta-\gamma)\right)}\right. \\
& \left.\left[D\left(w_{n-1}, T w_{n-1}\right)+D\left(w_{n}, T w_{n}\right)\right]^{\left(p \widehat{w}^{2} /(\widehat{w}-\beta)(\widehat{w}-\gamma)\right)} \cdot\left[D\left(w_{n-1}, T w_{n}\right)+D\left(w_{n}, T w_{n-1}\right)\right]^{\left(p \gamma^{2} /(\gamma-\beta)(\gamma-\widehat{w})\right)}\right] \\
& =\lambda \max \left[D\left(w_{n-1}, w_{n}\right)^{p} \cdot D\left(w_{n-1}, w_{n}\right)^{\left(p \beta^{2} /(\beta-\widehat{w})(\beta-\gamma)\right)} \cdot D\left(w_{n}, w_{n+1}\right)^{\left(p \beta^{2} /(\beta-\widehat{w})(\beta-\gamma)\right)} .\right. \\
& \left.\left[D\left(w_{n-1}, w_{n}\right)+D\left(w_{n}, w_{n+1}\right)\right]^{\left(\hat{w}^{2} /(\widehat{w}-\beta)(\widehat{w}-\gamma)\right)} \cdot\left[D\left(w_{n-1}, w_{n+1}\right)+D\left(w_{n}, w_{n}\right)\right]^{\left(p \gamma^{2} /(\gamma-\beta)(\gamma-\widehat{w})\right)}\right] \\
& \leq \lambda \max \left[D\left(w_{n-1}, w_{n}\right)^{p} \cdot D\left(w_{n-1}, w_{n}\right)^{\left(p \beta^{2} /(\beta-\widehat{w})(\beta-\gamma)\right)} \cdot D\left(w_{n}, w_{n+1}\right)^{\left(p \beta^{2} /(\beta-\widehat{w})(\beta-\gamma)\right)} .\right. \\
& \left.\left[D\left(w_{n-1}, w_{n}\right)+D\left(w_{n}, w_{n+1}\right)\right]^{\left(p \hat{w}^{2} /(\widehat{w}-\beta)(\widehat{w}-\gamma)\right)} \cdot\left[D\left(w_{n-1}, w_{n}\right)+D\left(w_{n}, w_{n+1}\right)\right]^{\left(p \gamma^{2} /(\gamma-\beta)(\gamma-\widehat{w})\right)}\right] \\
& =\lambda \max \left[D\left(w_{n-1}, w_{n}\right)^{p} \cdot D\left(w_{n-1}, w_{n}\right)^{\left(p \beta^{2} /(\beta-\widehat{w})(\beta-\gamma)\right)} \cdot D\left(w_{n}, w_{n+1}\right)^{\left(p \beta^{2} /(\beta-\widehat{w})(\beta-\gamma)\right) .}\right. \\
& \left.\left[D\left(w_{n-1}, w_{n}\right)+D\left(w_{n}, w_{n+1}\right)\right]^{\left(p \widehat{w}^{2} /(\widehat{w}-\beta)(\widehat{w}-\gamma)\right)+\left(p \gamma^{2} /(\gamma-\beta)(\gamma-\widehat{w})\right)}\right] \\
& \leq \lambda \max \left[D\left(w_{n-1}, w_{n}\right)^{p} \cdot D\left(w_{n-1}, w_{n}\right)^{\left(p \beta^{2} /(\beta-\widehat{w})(\beta-\gamma)\right)} \cdot D\left(w_{n}, w_{n+1}\right)^{\left(p \beta^{2} /(\beta-\widehat{w})(\beta-\gamma)\right) .}\right. \\
& \left.\left[D\left(w_{n-1}, w_{n}\right) \cdot D\left(w_{n}, w_{n+1}\right)\right]\left(\hat{w}^{2} /(\widehat{w}-\beta)(\widehat{w}-\gamma)\right)+\left(p \gamma^{2} /(\gamma-\beta)(\gamma-\widehat{w})\right)\right] \\
& =\lambda \max \left[D\left(w_{n-1}, w_{n}\right)^{p}, D\left(w_{n-1}, w_{n}\right)^{p+\left(p \beta^{2} /(\beta-\widehat{w})(\beta-\gamma)\right)+\left(p \widehat{w}^{2} /(\widehat{w}-\beta)(\widehat{w}-\gamma)\right)+\left(p \gamma^{2} /(\gamma-\beta)(\gamma-\widehat{w})\right)}\right. \text {. } \\
& \left.D\left(w_{n}, w_{n+1}\right)\left(p \beta^{2} /(\beta-\widehat{w})(\beta-\gamma)\right)+\left(p \widehat{w}^{2} /(\widehat{w}-\beta)(\widehat{w}-\gamma)\right)+\left(p \gamma^{2} /(\gamma-\beta)(\gamma-\widehat{w})\right)\right] \\
& =\lambda \max \left\{D\left(w_{n-1}, w_{n}\right)^{p}, D\left(w_{n}, w_{n+1}\right)^{p}\right\} \text {. }
\end{aligned}
$$

Provided that $\max \left\{D\left(w_{n}, w_{n+1}\right), D\left(w_{n-1}, w_{n}\right)\right\}=D\left(w_{n}\right.$, $\left.w_{n+1}\right)$, at that time,

$$
D\left(w_{n}, w_{n+1}\right) \leq \lambda D\left(w_{n}, w_{n+1}\right),
$$

which is a contradiction. We deduce that

$$
D\left(w_{n}, w_{n+1}\right) \leq \lambda D\left(w_{n-1}, w_{n}\right) .
$$

We conclude that $\left\{D\left(w_{n-1}, w_{n}\right)\right\}$ is a nonincreasing sequence with nonnegative terms. As a result, there is a nonnegative constant $\rho$ such that $\lim _{n \rightarrow \infty} D\left(w_{n-1}\right.$, $\left.w_{n}\right)=\rho$. We shall indicate that $\rho>0$. Indeed, from (59), we derive that

$$
D\left(w_{n}, w_{n+1}\right) \leq \lambda D\left(w_{n-1}, w_{n}\right) \leq \lambda^{n} D\left(w_{0}, w_{1}\right) .
$$


Pause the proof and go behind the closing lines of Theorem 2.

Theorem 7. Consider an $\mathscr{F}$-complete $\mathscr{F}$-metric space $(A, D)$ and let $T$ be a fractional symmetric $\alpha-\eta$-contraction of type-III meeting the following assertions:

(i) $T$ is an $\alpha$-admissible mapping with respect to $\eta$

(ii) There exists $w_{0} \in A$ such that $\alpha\left(w_{0}, T w_{0}\right) \geq \eta$ $\left(w_{0}, T w_{0}\right)$

(iii) An iteration $\left\{w_{n}\right\}$ in $A$ is such that $\alpha\left(w_{n}, w_{n+1}\right)$ $\geq \eta\left(w_{n}, w_{n+1}\right)$ escorted by $w_{n} \longrightarrow w^{*}$ at the same time $n \longrightarrow \infty$; after that $\alpha\left(w_{n}, w^{*}\right) \geq \eta\left(w_{n}, w^{*}\right)$ holds for each $n \in \mathbb{N}$

Afterwards, $T$ possesses a fixed point in A.

Proof. Similar to the same lines of Theorem 3, considering (iii), $\alpha\left(w_{n}, w^{*}\right) \geq \eta\left(w_{n}, w^{*}\right)$ for all $n \in \mathbb{N}$. By $\left(D_{3}\right)$, we have

$$
f\left(D\left(T w^{*}, w^{*}\right)\right) \leq f\left(D\left(T w^{*}, T w_{n}\right)+D\left(w_{n}, w^{*}\right)\right)+\mu .
$$

Using (40) along with $\left(\mathscr{F}_{1}\right)$, we have

$$
\begin{aligned}
f\left(D\left(T w^{*}, w^{*}\right)^{p}\right) \leq & f\left(\left(D\left(T w^{*}, T w_{n}\right)^{p}\right)+D\left(T w_{n}, w^{*}\right)^{p}\right)+\mu \\
\leq & f\left(\lambda \left(D\left(w^{*}, w_{n}\right)^{p}, D\left(T w^{*}, w^{*}\right)^{\left(p \beta^{2} /(\beta-\widehat{w})(\beta-\gamma)\right)} \cdot D\left(w_{n}, T w_{n}\right)^{\left(p \beta^{2} /(\beta-\widehat{w})(\beta-\gamma)\right)}\right.\right. \\
& \cdot\left[D\left(T w^{*}, w^{*}\right)+D\left(w_{n}, T w_{n}\right)\right]^{\left(p \widehat{w}^{2} /(\widehat{w}-\beta)(\widehat{w}-\gamma)\right)} \\
& \left.\left.\cdot\left[D\left(T w_{n}, w^{*}\right)+D\left(w_{n}, T w^{*}\right)\right]^{\left(p \gamma^{2} /(\gamma-\beta)(\gamma-\widehat{w})\right)}+D\left(w_{n+1}, w^{*}\right)^{p}\right)\right)+\mu .
\end{aligned}
$$

Using (25) the factuality is

$\lim _{n \longrightarrow \infty} D\left(w_{n}, w^{*}\right)=0$ as long as $\lim _{n \longrightarrow \infty} D\left(w_{n+1}, w^{*}\right)=0$,

and we obtain

$$
f\left(D\left(w^{*}, T w^{*}\right)^{p}\right) \leq f\left(D\left(w^{*}, T w^{*}\right)^{p}\right)+\mu .
$$

Utilizing $\left(\mathscr{F}_{2}\right)$, we have

$$
\lim _{n \longrightarrow \infty} f\left(D\left(w^{*}, T w^{*}\right)\right)+\mu=-\infty,
$$

which is a logical inconsistency. Along these lines, $D\left(w^{*}, T w^{*}\right)=0$; that is, $w^{*}$ possesses a fixed point of $T$.

\section{Fractional Symmetric $\alpha-\eta$-Contraction of Type-IV}

In this part, we propose a new notion, fractional symmetric $\alpha$ - $\eta$-contraction of type-IV, in the framework of $\mathscr{F}$-complete $\mathscr{F}$-metric space. (i) $T$ is an $\alpha$-admissible mapping concerning $\eta$

(ii) There exists $w_{0} \in A$ which connotes that $\alpha\left(w_{0}, T w_{0}\right) \geq \eta\left(w_{0}, T w_{0}\right)$

(iii) $T$ is $\alpha-\eta$-continuous

Definition 10. Consider an $\mathscr{F}$-metric space $(A, D)$ with a self-map $T: A \longrightarrow A$ and two functions $\alpha, \eta: A \times A \longrightarrow$ $[0,+\infty)$. We say that $T$ is a fractional symmetric $\alpha$ - $\eta$-contraction type-IV along with constants $\lambda \in[0,1)$ and $\beta, \widehat{w}, \gamma \in(0,1)$ with $\beta+\widehat{w}+\gamma<1$ such that, whenever $\alpha(w, v) \geq \eta(w, v)$, we have

$$
D(T w, T v)^{p} \leq \lambda\left(\check{S}_{4}(w, v)\right),
$$

where

$$
\begin{aligned}
\check{S}_{4}(w, v)= & \lambda\left\{D(w, v)\left(p \beta^{3} /(\beta-\widehat{w})(\beta-\gamma)\right) \cdot D(w, T w)\left(p \beta^{3} /(\beta-\widehat{w})(\beta-\gamma)\right) \cdot[D(w, T w)+D(v, T v)]\left(p \widehat{w}^{3} /(\widehat{w}-\beta)(\widehat{w}-\gamma)\right)\right. \\
& \left.\cdot[D(w, T v)+D(v, T w)]\left(p \gamma^{3} /(\gamma-\beta)(\gamma-\widehat{w})\right)\right\},
\end{aligned}
$$

where $p \in[1, \infty)$, for all $w, v \in A \backslash \operatorname{Fix}(T)$.

Now we declare and demonstrate our next theorem.

Theorem 8. Let an $\mathscr{F}$-complete $(A, D)$ be an $\mathscr{F}$-metric space along with $T$ being a fractional symmetric $\alpha$ - $\eta$-contraction of type-IV that meets the following assertions:
After that, $T$ possesses a fixed point in $A$.

Proof. Consider $w_{0}$ in $A$ with the aim that $\alpha\left(w_{0}, T w_{0}\right) \geq \eta\left(w_{0}, T w_{0}\right)$. Take any $w_{0} \in A$; we build a chain $\left\{w_{n}\right\}_{n=1}^{\infty}$ in such a way that $w_{1}=T w_{0}$ and $w_{2}=T w_{1}=T^{2} w_{0}$. 
Proceeding with this exercise, $w_{n+1}=T w_{n}=T^{n+1} w_{0}$, for every $n \in \mathbb{N}$. As long as mapping $T$ is $\alpha$-admissible with respect to $\eta$, at that time $\alpha\left(w_{0}, w_{1}\right)=\alpha\left(w_{0}, T w_{0}\right) \geq$ $\eta\left(w_{0}, T w_{0}\right)=\eta\left(w_{0}, w_{1}\right)$. Carrying on in this way, we get $\alpha\left(w_{n-1}, w_{n}\right) \geq \eta\left(w_{n-1}, w_{n}\right)=\eta\left(w_{n-1}, T w_{n-1}\right), \quad$ each $n \in \mathbb{N}$.
Provided that $w_{n+1}=w_{n}$ for some $n \in \mathbb{N}$, then $w_{n}=w^{*}$ is a fixed point of $T$. So, we assume that $w_{n} \neq w_{n+1}$ accompanied by

$$
D\left(T w_{n-1}, T w_{n}\right)=D\left(w_{n}, T w_{n}\right)>0, \quad \text { for all } n \in \mathbb{N} .
$$

As $T$ is fractional symmetric $\alpha-\eta$-contraction of type-IV, a part of $n \in \mathbb{N}$, we have

$$
\begin{aligned}
& D\left(w_{n}, w_{n+1}\right)^{p}=D\left(T w_{n-1}, T w_{n}\right)^{p} \leq \lambda\left[D\left(w_{n-1}, w_{n}\right)^{\left(p \beta^{3} /(\beta-\widehat{w})(\beta-\gamma)\right)} .\right. \\
& D\left(w_{n-1}, T w_{n-1}\right)^{\left(p \beta^{3} /(\beta-\widehat{w})(\beta-\gamma)\right)} \cdot\left[D\left(w_{n-1}, T w_{n-1}\right)+D\left(w_{n}, T w_{n}\right)\right]^{\left(p \hat{w}^{3} /(\widehat{w}-\beta)(\widehat{w}-\gamma)\right)} \\
& \left.\cdot\left[D\left(w_{n-1}, T w_{n}\right)+D\left(w_{n}, T w_{n-1}\right)\right]^{\left(p \gamma^{3} /(\gamma-\beta)(\gamma-\widehat{w})\right)}\right] \\
& =\lambda\left[D\left(w_{n-1}, w_{n}\right)^{\left(p \beta^{3} /(\beta-\widehat{w})(\beta-\gamma)\right)} \cdot D\left(w_{n}, w_{n+1}\right)\right)^{\left(p \beta^{3} /(\beta-\widehat{w})(\beta-\gamma)\right)} . \\
& \left.\left[D\left(w_{n-1}, w_{n}\right)+D\left(w_{n}, w_{n+1}\right)\right]^{\left(p \widehat{w}^{3} /(\widehat{w}-\beta)(\widehat{w}-\gamma)\right)} \cdot\left[D\left(w_{n-1}, w_{n+1}\right)+D\left(w_{n}, w_{n}\right)\right]^{\left(p \gamma^{3} /(\gamma-\beta)(\gamma-\widehat{w})\right)}\right] \\
& \leq \lambda\left[D\left(w_{n-1}, w_{n}\right)^{\left(p \beta^{3} /(\beta-\widehat{w})(\beta-\gamma)\right)} \cdot D\left(w_{n}, w_{n+1}\right)^{\left(p \beta^{3} /(\beta-\widehat{w})(\beta-\gamma)\right)}\right. \text {. } \\
& \left.\left[D\left(w_{n-1}, w_{n}\right)+D\left(w_{n}, w_{n+1}\right)\right]^{\left(p \widehat{w}^{3} /(\widehat{w}-\beta)(\widehat{w}-\gamma)\right)} \cdot\left[D\left(w_{n-1}, w_{n}\right)+D\left(w_{n}, w_{n+1}\right)\right]^{\left(p \gamma^{3} /(\gamma-\beta)(\gamma-\widehat{w})\right)}\right] \\
& =\lambda\left[D\left(w_{n-1}, w_{n}\right)^{\left(p \beta^{3} /(\beta-\widehat{w})(\beta-\gamma)\right)} \cdot D\left(w_{n}, w_{n+1}\right)^{\left(p \beta^{3} /(\beta-\widehat{w})(\beta-\gamma)\right)} .\right. \\
& \left.\left[D\left(w_{n-1}, w_{n}\right)+D\left(w_{n}, w_{n+1}\right)\right]^{\left(p \gamma^{3} /(\gamma-\beta)(\gamma-\widehat{w})\right)}\right] \\
& \leq \lambda\left[D\left(w_{n-1}, w_{n}\right)^{\left(p \beta^{3} /(\beta-\widehat{w})(\beta-\gamma)\right)} \cdot D\left(w_{n}, w_{n+1}\right)^{\left(p \beta^{3} /(\beta-\widehat{w})(\beta-\gamma)\right)} .\right. \\
& \left.\left[D\left(w_{n-1}, w_{n}\right) \cdot D\left(w_{n}, w_{n+1}\right)\right]^{\left(p \widehat{w}^{3} /(\widehat{w}-\beta)(\widehat{w}-\gamma)\right)+\left(p \gamma^{3} /(\gamma-\beta)(\gamma-\widehat{w})\right)}\right] \\
& =\lambda\left[D\left(w_{n-1}, w_{n}\right)^{\left(p \beta^{3} /(\beta-\widehat{w})(\beta-\gamma)\right)+\left(p \hat{w}^{3} /(\widehat{w}-\beta)(\widehat{w}-\gamma)\right)+\left(p \gamma^{3} /(\gamma-\beta)(\gamma-\widehat{w})\right)}\right. \\
& \left.\cdot D\left(w_{n}, w_{n+1}\right)\left(p \beta^{3} /(\beta-\widehat{w})(\beta-\gamma)\right)+\left(\hat{w}^{3} /(\widehat{w}-\beta)(\widehat{w}-\gamma)\right)+\left(p \gamma^{3} /(\gamma-\beta)(\gamma-\widehat{w})\right)\right] \\
& =\lambda\left\{D\left(w_{n-1}, w_{n}\right) \cdot D\left(w_{n}, w_{n+1}\right)\right\}^{p(\beta+\widehat{w}+\gamma)} \\
& \leq \lambda \max \left\{D\left(w_{n-1}, w_{n}\right)^{p}, D\left(w_{n}, w_{n+1}\right)^{p}\right\} .
\end{aligned}
$$

On condition that $\max \left\{D\left(w_{n}, w_{n+1}\right), D\left(w_{n-1}, w_{n}\right)\right\}=$ $D\left(w_{n}, w_{n+1}\right)$, at that time,

$$
D\left(w_{n}, w_{n+1}\right) \leq \lambda D\left(w_{n}, w_{n+1}\right),
$$

which is a contradiction. We deduce that

$$
D\left(w_{n}, w_{n+1}\right) \leq \lambda D\left(w_{n-1}, w_{n}\right) .
$$

Let up the closing lines of Theorem 2.

Theorem 9. Consider an $\mathscr{F}$-complete $\mathscr{F}$-metric space $(A, D)$ and suppose that $T$ is a fractional symmetric $\alpha$ - $\eta$-contraction of type-IV fulfilling the accompanying affirmations:

(i) $T$ is an $\alpha$-admissible mapping concerning $\eta$ (ii) There exists $w_{0} \in A$ to such an extent that $\alpha\left(w_{0}, T w_{0}\right) \geq \eta\left(w_{0}, T w_{0}\right)$

(iii) An iteration $\left\{w_{n}\right\}$ in $A$ is analogous to $\alpha\left(w_{n}, w_{n+1}\right) \geq$ $\eta\left(w_{n}, w_{n+1}\right)$ escorted by $w_{n} \longrightarrow w^{*}$ at the same time $n \longrightarrow \infty$; after that $\alpha\left(w_{n}, w^{*}\right) \geq \eta\left(w_{n}, w^{*}\right)$ holds for each $n \in \mathbb{N}$

Afterwards, $T$ possesses a fixed point in A.

Whether $\eta(w, v)=1$, in Theorems 2, 3, 4, and 5, we introduce the following corollaries.

Corollary 1. Consider an $\mathscr{F}$-complete $\mathscr{F}$-metric space $(A, D)$ and suppose that $T$ is a fractional symmetric $\alpha$ - $\eta$-contraction of type-I fulfilling the accompanying affirmations: 
(i) $T$ is an $\alpha$-admissible mapping

(ii) There subsist $w_{0} \in A$ parallel to $\alpha\left(w_{0}, T w_{0}\right) \geq 1$

(iii) $T$ is $\alpha$ - $\eta$-continuous

Afterwards, $T$ possesses a fixed point in A.

Corollary 2. Consider an $\mathscr{F}$-complete $\mathscr{F}$-metric space $(A, D)$ and let $T$ be fractional symmetric $\alpha$ - $\eta$-contraction of type-I fulfilling the accompanying affirmations:

(i) $T$ is an $\alpha$-admissible mapping

(ii) There subsist $w_{0} \in A$ parallel to $\alpha\left(w_{0}, T w_{0}\right) \geq 1$

(iii) An iteration $\left\{w_{n}\right\}$ in $A$ is analogous to $\alpha\left(w_{n}, w_{n+1}\right) \geq$ 1 escorted by $w_{n} \longrightarrow w^{*}$ at the same time $n \longrightarrow \infty$; after that $\alpha\left(w_{n}, w^{*}\right) \geq 1$ holds for each $n \in \mathbb{N}$

Afterwards, $T$ possesses a fixed point in A.

Corollary 3. Let an $\mathscr{F}$-complete $(A, D)$ be $\mathscr{F}$-metric space and let $T$ be a fractional symmetric $\alpha-\eta$-contraction of type-II meeting the accompanying affirmations:

(i) $T$ is an $\alpha$-admissible mapping

(ii) There subsist $w_{0} \in A$ parallel to $\alpha\left(w_{0}, T w_{0}\right) \geq 1$

(iii) $T$ is $\alpha$ - $\eta$-continuous

Then $T$ gets a fixed point in $A$.
Corollary 4. Let an $\mathscr{F}$-complete $(A, D)$ be $\mathscr{F}$-metric space and let $T$ be a fractional symmetric $\alpha-\eta$-contraction of type-II meeting the accompanying affirmations:

(i) $T$ is an $\alpha$-admissible mapping

(ii) There subsist $w_{0} \in A$ parallel to $\alpha\left(w_{0}, T w_{0}\right) \geq 1$

(iii) An iteration $\left\{w_{n}\right\}$ in $A$ is analogous to $\alpha\left(w_{n}, w_{n+1}\right) \geq$ 1 escorted by $w_{n} \longrightarrow w^{*}$ at the same time $n \longrightarrow \infty$; after that $\alpha\left(w_{n}, w^{*}\right) \geq 1$ holds for each $n \in \mathbb{N}$

Afterwards, $T$ possesses a fixed point in A.

In similar fashion, we can deduce Corollaries 1, 2, 3, and 4 for fractional symmetric $\alpha-\eta$-contraction of type-III and that of type-IV, respectively.

\section{Consequences}

As a consequence of our results, we derive some effect for Suzuki-type fractional symmetric contractions and orbitally $T$-complete and orbitally continuous mappings in $\mathscr{F}$-metric spaces.

Theorem 10. Consider an $\mathscr{F}$-metric space $(A, D)$ and let $T$ be a continuous self-mapping on A. Assume that there exists $r \in[0,1)$ in addition to $\beta, \widehat{w}, \gamma \in(0,1)$ such that

$$
D(w, T w) \leq D(w, v) \text { implies } D(T w, T v)^{p} \leq r\left(\check{S}_{1}(w, v)\right),
$$

where

$$
\begin{aligned}
\check{S}_{1}(w, v)= & D(w, v)^{p} \cdot D(w, T w)^{(p /(\beta-\widehat{w})(\beta-\gamma))} \cdot D(v, T v)^{(p /(\beta-\widehat{w})(\beta-\gamma))} \cdot[D(w, T w)+D(v, T v)]^{(p /(\widehat{w}-\beta)(\widehat{w}-\gamma))} \\
& \cdot[D(w, T v)+D(v, T w)]^{(p /(\gamma-\beta)(\gamma-\widehat{w}))}
\end{aligned}
$$

where $p \in[1, \infty)$, for all $w, v \in A \backslash \operatorname{Fix}(T)$.

At that time, $T$ possesses a fixed point in $A$.

Proof. Describe $\alpha, \eta: A \times A \longrightarrow[0,+\infty)$ by

$$
\alpha(w, v)=D(w, v), \eta(w, v)=D(w, T w), \quad \text { for all } w, v \in A,
$$

and $\beta, \widehat{w}, \gamma \in(0,1)$, and $r \in[0,1)$. It is clear that

$$
\eta(w, v) \leq \alpha(w, v), \quad \text { for all } w, v \in A,
$$

which means that conditions (i)-(iii) of our Theorem 2 hold true. Let

$$
\eta(w, T w) \leq \alpha(w, v) \text { then } D(w, T w) \leq D(w, v),
$$

which implies contractive condition:

$$
D(T w, T v)^{p} \leq r\left(\check{S}_{1}(w, v)\right) .
$$

Finally, every constraint of Theorem 2 holds true. Hence, $T$ possesses a fixed point in $A$.

Theorem 11. Consider an $\mathscr{F}$-metric space $(A, D)$ and let $T$ be a self-mapping of $A$. Suppose that the following assertions hold:

(i) $(A, D)$ is an orbitally $T$-complete $\mathscr{F}$-metric space.

(ii) There exists $r \in[0,1)$ in addition to $\beta, \widehat{w}, \gamma \in(0,1)$ such that

$$
D(T w, T v)^{p} \leq r\left(\check{S}_{1}(w, v)\right),
$$

(i) where 


$$
\begin{aligned}
\check{S}_{1}(w, v)= & D(w, v)^{p} \cdot D(w, T w)^{(p /(\beta-\widehat{w})(\beta-\gamma))} \cdot D(v, T v)^{(p /(\beta-\widehat{w})(\beta-\gamma))} \cdot[D(w, T w)+D(v, T v)]^{(p /(\widehat{w}-\beta)(\widehat{w}-\gamma))} \\
& \cdot[D(w, T v)+D(v, T w)]^{(p /(\gamma-\beta)(\gamma-\widehat{w}))}
\end{aligned}
$$

(ii) $p \in[1, \infty)$, for all $w, v \in O(\omega)$ for some $\omega \in A$, where $O(\omega)$ is an orbit of $\omega$,

(iii) if $\left\{v_{n}\right\}$ is a sequence such that $\left\{v_{n}\right\} \subseteq O(\omega)$ with $v_{n} \longrightarrow v^{*}$ as $n \longrightarrow \infty$, then $v^{*} \in O(\omega)$.

Then, $T$ possesses a fixed point.

Proof. Describe $\alpha, \eta: A \times A \longrightarrow[0,+\infty)$, by $\alpha(w, v)=3$ on $O(\omega) \times O(\omega)$ and $\alpha(w, v)=0$; otherwise, $\eta(w, v)=1$ for all $w, v \in A$ (see Remark 6 [11]). Then $(A, D)$ is an $\alpha$ - $\eta$-complete $\mathscr{F}$-metric and $T$ is an $\alpha$-admissible mapping with respect to $\eta$. Let $\alpha(w, v) \geq \eta(w, v)$; then $w, v \in O(\omega)$, and then, from (ii), we have

$$
D(T w, T v)^{p} \leq r\left(\check{S}_{1}(w, v)\right),
$$

where

$$
\begin{aligned}
\check{S}_{1}(w, v)= & D(w, v)^{p} \cdot D(w, T w)^{(p /(\beta-\widehat{w})(\beta-\gamma))} \cdot D(v, T v)^{(p /(\beta-\widehat{w})(\beta-\gamma))} \cdot[D(w, T w)+D(v, T v)]^{(p /(\widehat{w}-\beta)(\widehat{w}-\gamma))} . \\
& {[D(w, T v)+D(v, T w)]^{(p /(\gamma-\beta)(\gamma-\widehat{w}))} . }
\end{aligned}
$$

That is, $T$ is a fractional symmetric $\alpha-\eta$-contraction of type-I. Let $\left\{v_{n}\right\}$ be a sequence commensurate with $\alpha\left(v_{n}\right.$, $\left.v_{n+1}\right) \geq \eta\left(v_{n}, v_{n+1}\right)$ together with $v_{n} \longrightarrow v^{*}$ for $n \longrightarrow \infty$. So, $\left\{v_{n}\right\} \subseteq O(\omega)$. From (iii), $v^{*} \in O(\omega)$; that is, $\alpha\left(v_{n}, v^{*}\right) \geq$ $\eta\left(v_{n}, v^{*}\right)$. Hence, every norm of Theorem 3 holds true. Thus, $T$ possesses a fixed point.

Theorem 12. Consider an $\mathscr{F}$-metric space $(A, D)$ and let $T$ be a self-mapping of $A$. Suppose that the following assertions hold: (i) For all $w, v \in O(\omega)$, there exists $r \in[0,1)$ along with $\beta, \widehat{w}, \gamma \in(0,1)$, such that

$$
D(T w, T v)^{p} \leq r\left(\check{S}_{1}(w, v)\right),
$$

(ii) where

$$
\begin{aligned}
\check{S}_{1}(w, v)= & D(w, v)^{p} \cdot[D(w, T w)]^{(p /(\beta-\widehat{w})(\beta-\gamma))} \cdot[D(v, T v)]^{(p /(\beta-\widehat{w})(\beta-\gamma))} \cdot[D(w, T w)+D(v, T v)]^{(p /(\widehat{w}-\beta)(\widehat{w}-\gamma))} \\
& \cdot[D(w, T v)+D(v, T w)]^{(p /(\gamma-\beta)(\gamma-\widehat{w}))}
\end{aligned}
$$

(iii) $p \in[1, \infty)$, for some $\omega \in A$,

(iv) the operator $T$ is orbitally continuous.

Afterwards, $T$ possesses a fixed point.

Proof. Describe $\alpha, \eta: A \times A \longrightarrow[0,+\infty)$, by $\alpha(w, v)=3$ on $O(\omega) \times O(\omega)$ and $\alpha(w, v)=0$; otherwise, $\eta(w, v)=1$ (see Remark 1.1 [12]), and we know that $T$ is an $\alpha-\eta$-continuous mapping. Let $\alpha(w, v) \geq \eta(w, v)$; then $w, v \in O(\omega)$. So $T w, T v \in O(\omega)$; that is, $\alpha(T w, T v) \geq \eta(T w, T v)$. Therefore, $T$ is an $\alpha$-admissible mapping with respect to $\eta$. From (i), we have

$$
D(T w, T v)^{p} \leq r\left(\check{S}_{1}(w, v)\right)
$$

where

$$
\begin{aligned}
\check{S}_{1}(w, v)= & D(w, v)^{p} \cdot D(w, T w)^{(p /(\beta-\widehat{w})(\beta-\gamma))} \cdot D(v, T v)^{(p /(\beta-\widehat{w})(\beta-\gamma))} \cdot[D(w, T w)+D(v, T v)]^{(p /(\widehat{w}-\beta)(\widehat{w}-\gamma))} . \\
& {[D(w, T v)+D(v, T w)]^{(p /(\gamma-\beta)(\gamma-\widehat{w}))} }
\end{aligned}
$$

and aforesaid $T$ is a fractional symmetric $\alpha-\eta$-contraction of type-I. Hence, each constraint of Theorem 2 holds true. Thus, $T$ gets a fixed point.
Theorem 13. Consider an $\mathscr{F}$-metric space $(A, D)$ and let $T$ be a self-mapping of $A$. Suppose that the following assertions hold: 
(i) $(A, D)$ is an orbitally $T$-complete $\mathscr{F}$-metric space;

(ii) there subsist $r \in[0,1)$ parallel to $\beta, \widehat{w}, \gamma \in(0,1)$ such $D(T w, T v)^{p} \leq r\left(\check{S}_{2}(w, v)\right)$ that

(iii) where

$$
\begin{aligned}
\check{S}_{2}(w, v)= & D(w, v)^{p} \cdot D(w, T w)^{(p \beta /(\beta-\widehat{w})(\beta-\gamma))} \cdot D(v, T v)^{(p \beta /(\beta-\widehat{w})(\beta-\gamma))} \cdot[D(w, T w)+D(v, T v)]^{(p \widehat{w} /(\widehat{w}-\beta)(\widehat{w}-\gamma))} \\
& {[D(w, T v)+D(v, T w)]^{(p \gamma /(\gamma-\beta)(\gamma-\widehat{w}))} }
\end{aligned}
$$

(iv) $p \in[1, \infty)$, for all $w, v \in O(\omega)$ for some $\omega \in A$, where $O(\omega)$ is an orbit of $\omega$;

(v) if $\left\{v_{n}\right\}$ is a sequence such that $\left\{v_{n}\right\} \subseteq O(\omega)$ with $v_{n} \longrightarrow v^{*}$ as $n \longrightarrow \infty$, then $v^{*} \in O(\omega)$.

Then, $T$ possesses a fixed point.

Theorem 14. Consider an $\mathscr{F}$-metric space $(A, D)$ and let $T$ be a self-mapping of $A$. Suppose that the following assertions hold:

$$
\begin{aligned}
\check{S}_{2}(w, v)= & D(w, v)^{p} \cdot D(w, T w)^{(p \beta /(\beta-\widehat{w})(\beta-\gamma))} \cdot D(v, T v)^{(p \beta /(\beta-\widehat{w})(\beta-\gamma))} \cdot[D(w, T w)+D(v, T v)]^{(p w /(\widehat{w}-\beta)(\widehat{w}-\gamma))} \\
& {[D(w, T v)+D(v, T w)]^{(p \gamma /(\gamma-\beta)(\gamma-\widehat{w}))} }
\end{aligned}
$$

(iii) $p \in[1, \infty)$, for some $\omega \in A$;

(iv) the operator $T$ is orbitally continuous.

Afterwards, $T$ possesses a fixed point.

Theorems 10, 11, and 12 can be derived easily for fractional symmetric contraction of type-III and that of type-IV, respectively.

\section{Application to Fractional-Order Differential Equations}

The local and nonlocal fractional differential equations have been recently proved to be significant tools in the modeling of many phenomena in numerous fields of science and building. The fractional-order differential equations have numerous applications in viscoelasticity, electrochemistry, control, porous media, electromagnetism, and so forth. For more details, see [2,13-19]. Our aim is to give the existence and uniqueness of bounded solution of local fractional-order differential equation given in (93). Consider a function $f:(0, \infty) \longrightarrow \mathbb{R}$. The conformable derivative of order $\alpha$ of $f$ at $t>0$ is defined by [20]

$$
D^{\alpha} f(t)=\lim _{\varepsilon \longrightarrow 0} \frac{f\left(t+\varepsilon t^{1-\alpha}\right)-f(t)}{\varepsilon} .
$$

(i) for all $w, v \in O(\omega)$, there subsist $r \in[0,1)$ along with $\beta, \widehat{w}, \gamma \in(0,1)$, such that

$$
D(T w, T v)^{p} \leq r\left(\check{S}_{2}(w, v)\right),
$$

(ii) where
The conformable fractional integral associated with (91) is defined by $[20,21]$

$$
I_{0}^{\alpha} f(t)=\int_{0}^{t} s^{\alpha-1} f(s) \mathrm{d} s .
$$

We consider the following boundary value problem of a conformable fractional-order differential equation:

$$
\begin{array}{r}
D^{\alpha} w(t)=\lambda f(t, w(t)), \quad t \in(0,1), 1<\alpha<2, \\
\text { with } w(0)=0, w(1)=\int_{0}^{1} w(s) \mathrm{d} s .
\end{array}
$$

The integral representation of the solution to the boundary value problem (93) is

$$
w(t)=\lambda \int_{0}^{1} G(t, s) f(s, w(s)) \mathrm{d} s,
$$

where $G(t, s)$ is a Green's function defined by

$$
G(t, s)= \begin{cases}-2 t s^{\alpha}+s^{\alpha-1}, & 0 \leq s \leq t \leq 1, \\ -2 t s^{\alpha}, & 0 \leq t \leq s \leq 1,\end{cases}
$$

and $\int_{0}^{1} w(s) \mathrm{d} s$ denotes the Riemann integrable of $w$ with respect to $s$ and $f:[0,1] \times \mathbb{R} \longrightarrow \mathbb{R}$ is a continuous function. 


$$
\begin{aligned}
w(t) & =c_{0}+c_{1} t+\lambda \int_{0}^{t} s^{\alpha-1} f(s, w(s)) \mathrm{d} s, \\
w(0) & =0 \Rightarrow c_{0}=0, \\
w(1) & =c_{1}+\lambda \int_{0}^{1} s^{\alpha-1} f(s, w(s)) \mathrm{d} s, \\
\int_{0}^{1} w(s) \mathrm{d} s & =\int_{0}^{1} c_{1} s \mathrm{~d} s+\lambda \int_{0}^{1} \int_{0}^{s} z^{\alpha-1} f(z, w(z)) \mathrm{d} z \mathrm{~d} s \\
& =\frac{1}{2} c_{1}+\lambda \int_{0}^{1} \int_{z}^{1} z^{\alpha-1} f(z, w(z)) \mathrm{d} s \mathrm{~d} z \\
& =\frac{1}{2} c_{1}+\lambda \int_{0}^{1}(1-z) z^{\alpha-1} f(z, w(z)) \mathrm{d} s \mathrm{~d} z \\
c_{1} & =-2 \lambda \int_{0}^{1} s^{\alpha} f(s, w(s)) \mathrm{d} s . \\
& =\frac{1}{2} c_{1}+\lambda \int_{0}^{1}\left(s^{\alpha-1}-s^{\alpha}\right) f(s, w(s)) \mathrm{d} s, \\
\frac{1}{2} c_{1} & =-\lambda \int_{0}^{1} s^{\alpha-1} f(s, w(s)) \mathrm{d} s+\lambda \int_{0}^{1}\left(s^{\alpha-1}-s^{\alpha}\right) f(s, w(s)) \mathrm{d} s \\
& =-\lambda \int_{0}^{1} s^{\alpha} f(s, w(s)) \mathrm{d} s \\
w & \\
w &
\end{aligned}
$$

So,

$$
\begin{aligned}
w(t) & =-2 t \lambda \int_{0}^{1} s^{\alpha} f(s, w(s)) \mathrm{d} s++\lambda \int_{0}^{t} s^{\alpha-1} f(s, w(s)) \mathrm{d} s \\
& =-2 t \lambda \int_{0}^{t} s^{\alpha} f(s, w(s)) \mathrm{d} s-2 t \lambda \int_{t}^{1} s^{\alpha} f(s, w(s)) \mathrm{d} s+\lambda \int_{0}^{t} s^{\alpha-1} f(s, w(s)) \mathrm{d} s \\
& =\lambda \int_{0}^{t}\left(-2 t s^{\alpha}+s^{\alpha-1}\right) f(s, w(s)) \mathrm{d} s+\lambda \int_{t}^{1}\left(-2 t s^{\alpha}\right) f(s, w(s)) \mathrm{d} s \\
& =\lambda \int_{0}^{1} G(t, s) f(s, w(s)) \mathrm{d} s .
\end{aligned}
$$

Let $C(I)$ be the linear space of all continuous functions defined on $I=[0,1]$, and let $D(w, v)=\|w-v\|_{\infty}=$ $\max _{t \in I}|w(t)-v(t)|$ for all $w, v \in C(I)$. Then, $(C(I), D)$ is an $\mathscr{F}$-complete metric space.
We consider the following conditions:

(a) There exists $r \in[0,1)$, and $\zeta: \mathbb{R}^{2} \longrightarrow \mathbb{R}$ is a function for each $a, b \in \mathbb{R}$ with $\zeta(a, b) \geq \xi(a, b)$, such that

$$
\begin{gathered}
|f(s, w(s)) \mathrm{d} s-f(s, v(s)) \mathrm{d} s| \leq r|w(s)-v(s)| \cdot|w(s)-T w(s)|^{(\beta /(\beta-\widehat{w})(\beta-\gamma))} \cdot|v(s)-T v(s)|^{(\beta /(\beta-\widehat{w})(\beta-\gamma))} \\
\cdot[|w(s)-T w(s)|+|v(s)-T v(s)|]^{(\widehat{w} /(\widehat{w}-\beta)(\widehat{w}-\gamma))} \cdot[|w(s)-T v(s)|+|v(s)-T w(s)|]^{(\gamma /(\gamma-\beta)(\gamma-\widehat{w}))},
\end{gathered}
$$


where $\beta, \widehat{w}, \gamma \in(0,1)$.

(b) There exists $w_{1} \in C(I)$ such that

$$
\begin{array}{r}
\zeta\left(w_{1}(t), \int_{0}^{1} G(t, s) f\left(s, w_{1}(s)\right) \mathrm{d} s\right) \\
\geq \xi\left(w_{1}(t), \int_{0}^{1} G(t, s) f\left(s, w_{1}(s)\right) \mathrm{d} s\right),
\end{array}
$$

for all $t \in I$;

(c) For each $w, v \in C(I)$, there exists $w_{1}, v_{1} \in C(I)$ such that

$$
\begin{aligned}
\zeta(w(t), v(t)) & \geq \xi(w(t), v(t)) \operatorname{implies} \zeta\left(\int_{0}^{1} G(t, s) f\left(s, w_{1}(s)\right) \mathrm{d} s, \int_{0}^{1} G(t, s) f\left(s, v_{1}(s)\right) \mathrm{d} s\right) \\
& \geq \xi\left(\int_{0}^{1} G(t, s) f\left(s, w_{1}(s)\right) \mathrm{d} s, \int_{0}^{1} G(t, s) f\left(s, v_{1}(s)\right) d s\right)
\end{aligned}
$$

for all $t \in I$;

(d) For any cluster point $w$ of a sequence $\left\{w_{n}\right\}$ of points in $C(I)$ with

$$
\begin{aligned}
\zeta\left(w_{n}, w_{n+1}\right) & \geq \xi\left(w_{n}, w_{n+1}\right), \\
\lim _{n \longrightarrow \infty} \inf \zeta\left(w_{n}, w\right) & \geq \lim _{n \longrightarrow \infty} \inf \xi\left(w_{n}, w\right) .
\end{aligned}
$$

Theorem 15. Suppose that conditions (a)-(d) are satisfied. Then, (93) has at least one solution $w^{*} \in C(I)$.
Proof. We know that $w \in C(I)$ is a solution of (93) if and only if $w \in C(I)$ is a solution of the fractional-order integral equation

$$
w(t)=\lambda \int_{0}^{1} G(t, s) f(s, w(s)) \mathrm{d} s, \quad \text { for all } \lambda, t \in I .
$$

We define a map $T: C(I) \longrightarrow C(I)$ by

$T w(t)=\lambda \int_{0}^{1} G(t, s) f(s, w(s)) \mathrm{d} s, \quad$ for all $t \in I$.

Then, problem (93) is equivalent to finding $w^{*} \in C(I)$, that is, a fixed point of $T$. Let $w, v \in C(I)$, such that $\zeta(w(t), v(t)) \geq 0$, for all $t \in I$. For using (a), we get

$$
\begin{aligned}
|T w(t)-T v(t)|= & \left|\lambda \int_{0}^{1} G(t, s)[f(s, w(s)) \mathrm{d} s-f(s, v(s))] \mathrm{d} s\right| \\
\leq & |\lambda| \int_{0}^{1} G(t, s)|f(s, w(s)) \mathrm{d} s-f(s, v(s)) \mathrm{d} s| \\
\leq & |\lambda| \int_{0}^{1} G(t, s) r \mathrm{~d} s|w(s)-v(s)| \cdot|w(s)-T w(s)|^{(\beta /(\beta-\widehat{w})(\beta-\gamma))} \cdot|v(s)-T v(s)|^{(\beta /(\beta-\widehat{w})(\beta-\gamma))} \\
& \cdot[|w(s)-T w(s)|+|v(s)-T v(s)|]^{(\widehat{w} /(\widehat{w}-\beta)(\widehat{w}-\gamma))} \cdot[|w(s)-T v(s)|+|v(s)-T w(s)|](\gamma /(\gamma-\beta)(\gamma-\widehat{w})) \\
\leq & \max _{t \in I} \int_{0}^{1} G(t, s) \mathrm{d} s r\|w(s)-v(s)\|_{\infty} \cdot\|w(s)-T w(s)\|_{\infty}^{(\beta /(\beta-\widehat{w})(\beta-\gamma))} \cdot\|v(s)-T v(s)\|_{\infty}^{(\beta /(\beta-\widehat{w})(\beta-\gamma))} \\
& \cdot\left[\|w(s)-T w(s)\|_{\infty}+\|v(s)-T v(s)\|_{\infty}\right]^{(\widehat{w} /(\widehat{w}-\beta)(\widehat{w}-\gamma))} \cdot\left[\|w(s)-T v(s)\|_{\infty}+\|v(s)-T w(s)\|_{\infty}\right]^{(\gamma /(\gamma-\beta)(\gamma-\widehat{w}))} \\
\leq & r\|w(s)-v(s)\|_{\infty} \cdot\|w(s)-T w(s)\|_{\infty}^{(\beta /(\beta-\widehat{w})(\beta-\gamma))} \cdot\|v(s)-T v(s)\|_{\infty}^{(\beta /(\beta-\widehat{w})(\beta-\gamma))} \cdot\|w(s)-T v(s)\|_{\infty}^{(p \gamma-q \gamma)} \\
& \cdot\left[\|w(s)-T w(s)\|_{\infty}+\|v(s)-T v(s)\|_{\infty}\right]^{(\widehat{w} /(\widehat{w}-\beta)(\widehat{w}-\gamma))} \cdot\left[\|w(s)-T v(s)\|_{\infty}+\|v(s)-T w(s)\|_{\infty}\right]^{(\gamma /(\gamma-\beta)(\gamma-\widehat{w}))} .
\end{aligned}
$$


Thus,

$$
\begin{aligned}
D(T w, T v) & <\left\{|w(s)-v(s)| \cdot|w(s)-T w(s)|^{(\beta /(\beta-\widehat{w})(\beta-\gamma))} \cdot|v(s)-T v(s)|^{(\beta /(\beta-\widehat{w})(\beta-\gamma))}\right. \\
\cdot & {\left.[|w(s)-T w(s)|+|v(s)-T v(s)|]^{(\widehat{w} /(\widehat{w}-\beta)(\widehat{w}-\gamma))} \cdot[|w(s)-T v(s)|+|v(s)-T w(s)|]^{(\gamma /(\gamma-\beta)(\gamma-\widehat{w}))}\right\} }
\end{aligned}
$$

for all $w, v \in C(I)$ such that $\zeta(w(t), v(t)) \geq \xi(w(t), v(t))$ for all $t \in I$. We define $\alpha: C(I) \times C(I) \longrightarrow[0, \infty)$ by

$$
\begin{aligned}
& \alpha(w, v)=\left\{\begin{array}{cc}
1, & \text { if } \zeta(w(t), v(t)) \geq 0, t \in I, \\
0, & \text { otherwise }
\end{array}\right\}, \\
& \eta(w, v)=\left\{\begin{array}{ll}
\frac{1}{2}, & \text { if } \xi(w(t), v(t)) \geq 0, t \in I, \\
0, & \text { otherwise }
\end{array}\right\} .
\end{aligned}
$$

$$
\begin{aligned}
D(T w, T v) \leq & r|w(s)-v(s)| \cdot|w(s)-T w(s)|^{(\beta /(\beta-\widehat{w})(\beta-\gamma))} \cdot|v(s)-T v(s)|^{(\beta /(\beta-\widehat{w})(\beta-\gamma))} \\
\cdot & {[|w(s)-T w(s)|+|v(s)-T v(s)|]^{(\widehat{w} /(\widehat{w}-\beta)(\widehat{w}-\gamma))} \cdot[|w(s)-T v(s)|+|v(s)-T w(s)|]^{(\gamma /(\gamma-\beta)(\gamma-\widehat{w}))} . }
\end{aligned}
$$

Obviously, $\alpha(w, v) \geq \eta(w, v)$ for all $w, v \in C(I)$. If $\alpha(w$, $v) \geq \eta(w, v)$ for each $w, v \in C(I)$, then $\zeta(w(t), v(t)) \geq$ $\xi(w(t), v(t))$. From (c), we have $\zeta(T w(t), T v(t)) \geq$ $\xi(T w(t), T v(t))$ and so $\alpha(T w, T v) \geq \eta(T w, T v)$. Thus, $T$ is an $\alpha$-admissible map concerning $\eta$. From (b), there subsist $w_{1} \in C(I)$ parallel to $\alpha\left(w_{1}, T w_{1}\right)=\eta\left(w_{1}, T w_{1}\right)$. By (d), we have that, for any cluster point $w$ of a sequence $\left\{w_{n}\right\}$ of points in $C(I)$ with $\alpha\left(w_{n}, w_{n+1}\right)=\eta\left(w_{n}, w_{n+1}\right), \lim _{n \longrightarrow \infty}$ $\inf \alpha\left(w_{n}, w\right)=\lim _{n \longrightarrow \infty} \inf \eta\left(w_{n}, w\right)$. By applying Theorem 2 , if $T$ has a fixed point in $C(I)$, there exists $w^{*} \in C(I)$ such that $T w^{*}=w^{*}$, and $w^{*}$ is a solution of (93).

7.1. Applications. The fractional-order differential equations emerge in various areas of engineering and scientific disciplines as the mathematical modeling of systems and processes in the fields of physics, chemistry, control theory, biology, economics, blood flow phenomena, signal and image processing, biophysics, aerodynamics, and fitting of experimental data.

7.2. Open Problem. What are the conditions for making a power of the contraction a nonnegative real number for fixed point and coincidence fixed point for two or more maps in various spaces?

\section{Conclusion}

The aim of this paper is to produce four new classes of type contractions. This research focuses on new idea of fractional symmetric $\alpha-\eta$-contractions of type-I, type-II, type-III, and type-IV in the structure of $\mathscr{F}$-metric space, which is different from and more general than ordinary metric. This paper will open a new conspiracy of fractional fixed-point theory. We develop here Suzuki-type fixed point results in orbitally complete $\mathscr{F}$-metric space. These new investigations and applications would enhance the impact of new setup.

\section{Data Availability}

The data used to support the findings of this study are available upon request.

\section{Conflicts of Interest}

The author declares that there are no conflicts of interest.

\section{References}

[1] W. A. Kirk, S. Reich, and P. veeramani, "Proximinal retracts and best proximity pair theorems," Numerical Functional Analysis and Optimization, vol. 24, no. 7-8, pp. 851-862, 2003.

[2] S. Reich, "Some problems and results in fixed point theory," Topological Methods in Nonlinear Functional Analysis, vol. 21, pp. 179-187, 1983.

[3] M. Abbas and T. Nazir, "Common fixed point of a power graphic contraction pair in partial metric spaces endowed with a graph," Fixed Point Theory and Applications, vol. 2013, Article ID 20, 2013.

[4] E. Karapinar, R. Agarwal, and H. Aydi, "Interpolative reich-rus-cirić type contractions on partial metric spaces," Mathematics, vol. 6, no. 11, p. 256, 2018.

[5] M. Jleli and B. Samet, "On a new generalization of metric spaces," Journal of Fixed Point Theory and Applications, vol. 20, no. 3, p. 128, 2018. 
[6] B. Samet, C. Vetro, and P. Vetro, "Fixed point theorems for Alpha-Psi -contractive type mappings," Nonlinear Analysis, vol. 75, pp. 215-216, 2012.

[7] B. Samet, C. Vetro, and P. Vetro, "Fixed point theorems for -contractive type mappings," Nonlinear Analysis: Theory, Methods \& Applications, vol. 75, no. 4, pp. 2154-2165, 2012.

[8] N. Hussain and P. Salimi Salimi, "Suzuki-wardowski type fixed point theorems for \$ \alpha-gf-contractions," Taiwanese Journal of Mathematics, vol. 18, no. 6, 2014.

[9] E. Karapinar and B. Samet, "Generalized contractive type mappings and related fixed point theorems with applications," Abstract and Applied Analysis, vol. 2012, Article ID 793486, 17 pages, 2012.

[10] MA. Kutbi, M. Arshad, and A. Hussain, "On modified contractive mappings," Abstract and Applied Analysis, vol. 2014, Article ID 657858, 7 pages, 2014.

[11] N. Hussain, M. A. Kutbi, and P. Salimi, "Fixed point theory in -complete metric spaces with applications," Abstract and Applied Analysis, vol. 2014, Article ID 280817, 11 pages, 2014.

[12] N. Hussain, A. Latif, and I. Iqbal, "Fixed point results for generalized F-contractions in modular metric and fuzzy metric spaces," Fixed Point Theory and Applications, vol. 2015, Article ID 158, 2015.

[13] R. S. Adiguzel, U. Aksoy, E. Karapinar, and I. M. Erhan, "On the solution of a boundary value problem associated with a fractional differential equation mathematical methods in the applied sciences," Mathematical Methods in the Applied Sciences, 2020.

[14] B. Alqahtani, H. Aydi, E. Rakočević, and V. Rakocevic, "A solution for volterra fractional integral equations by hybrid contractions," Mathematics, vol. 7, no. 8, p. 694, 2019.

[15] D. Baleanu, R. P. Agarwal, H. Mohammadi, and S. Rezapour, "Some existence results for a nonlinear fractional differential equationon partially ordered Banach spaces," Boundary Value Problems, vol. 112, p. 8, 2013.

[16] L. He, X. Dong, Z. Bai, and B. Chen, "Solvability of some twopoint fractional boundary value problems under barrier strip conditions," Journal of Function Spaces, vol. 2017, Article ID 1465623, 6 pages, 2017.

[17] A. Hussain, "Fractional convex type contraction with solution of fractional differential equation," AIMS Mathematics, vol. 5, no. 5, pp. 5364-5380, 2020.

[18] F. Jarad, T. Abdeljawad, and Z. Hammouch, "On a class of ordinary differential equations in the frame of AtanganaBaleanu fractional derivative," Chaos, Solitons \& Fractals, vol. 117, pp. 16-20, 2018.

[19] K. S. Miller and B. Ross, An Introduction to the Fractional Calculus and Fractional Differential Eqautions, John Wiley \& Sons, Hoboken, NJ, USA, 1993.

[20] R. Khalil, M. Al Horani, A. Yousef, and M. Sababheh, "A new definition of fractional derivative," Journal of Computational and Applied Mathematics, vol. 264, pp. 65-70, 2014.

[21] T. Abdeljawad, "On conformable fractional calculus," Journal of Computational and Applied Mathematics, vol. 279, pp. 57-66, 2015. 